\title{
WELL-POSEDNESS AND LONG TIME BEHAVIOR OF A PARABOLIC-HYPERBOLIC PHASE-FIELD SYSTEM WITH SINGULAR POTENTIALS
}

\author{
Maurizio Grasselli ${ }^{1}$, Alain Miranville ${ }^{2}$, \\ Vittorino Pata ${ }^{1}$ and Sergey Zelik ${ }^{2}$ \\ ${ }^{1}$ Politecnico di Milano \\ Dipartimento di Matematica "F. Brioschi" \\ Via E. Bonardi, 9 - 20133 Milano - Italy \\ ${ }^{2}$ Université de Poitiers \\ Laboratoire de Mathématiques et Applications \\ UMR CNRS 6086 - SP2MI \\ Boulevard Marie et Pierre Curie - Téléport 2 \\ 86962 Chasseneuil Futuroscope Cedex - France
}

\begin{abstract}
In this article, we study the long time behavior of a phase-field parabolichyperbolic system arising from the phase-field theory of phase transitions. This system consists of a parabolic equation governing the (relative) temperature which is nonlinearly coupled with a weakly damped semilinear hyperbolic equation ruling the evolution of the order parameter. The latter is a singular perturbation through an inertial term of the parabolic Allen-Cahn equation and it is characterized by the presence of a singular potential, e.g., of logarithmic type, instead of the classical double-well potential. We first prove the existence and uniqueness of strong solutions when the inertial coefficient $\varepsilon$ is small enough. Then, we construct a robust family of exponential attractors (as $\varepsilon$ goes to 0 ).
\end{abstract}

\section{INTRODUCTION}

We consider the following parabolic-hyperbolic system in a bounded smooth domain $\Omega \subset \mathbb{R}^{3}:$

$$
\left\{\begin{array}{l}
\partial_{t}(\theta+H(u))-\Delta_{x} \theta=0,\left.\quad \theta\right|_{\partial \Omega}=0,\left.\quad \theta\right|_{t=0}=\theta_{0}, \\
\varepsilon \partial_{t}^{2} u+\partial_{t} u-\Delta_{x} u+f(u)-H^{\prime}(u) \theta=g,\left.\quad u\right|_{\partial \Omega}=0,\left.\quad \xi_{u}\right|_{t=0}=\xi^{0},
\end{array}\right.
$$

1991 Mathematics Subject Classification. 35B40, 35B45, 37L25, 80A22.

Key words and phrases. Phase-field systems, hyperbolic equations, strong solutions, robust exponential attractors. 
where $u=u(t, x)$ and $\theta=\theta(t, x)$ are unknown functions, $\xi_{u}(t):=\left(u(t), \partial_{t} u(t)\right)$, $\varepsilon>0$ is a small parameter, $f$ and $H$ are given functions, $\Delta_{x}$ is the Laplacian with respect to $x=\left(x^{1}, x^{2}, x^{3}\right)$ and $g=g(x)$ are given external forces.

The study of such systems is motivated by the study of phase-field models (see $[\mathrm{BrS}]$ and the references therein). In this context, $\theta$ stands for the (relative) temperature and $u$ is an order parameter (or phase-field). This system was actually proposed as a (singular) perturbation of the classical phase-field model of Caginalp type corresponding to $\varepsilon=0$ in $(0.1)_{2}$ (see $[\mathrm{Ca}]$ ). From a physical point of view, equation $(0.1)_{2}$ can be justified as follows (for simplicity, we take $g \equiv 0$ ). When $\epsilon=0$, we can rewrite this equation in the form

$$
\frac{\partial u}{\partial t}=-\frac{\delta \mathcal{F}}{\delta u}
$$

where $\frac{\delta \mathcal{F}}{\delta u}$ denotes the variational derivative of the free energy

$$
\mathcal{F}=\frac{1}{2}|\nabla u|^{2}+F(u)-H(u) \theta
$$

with respect to the order parameter (here, $F$ is a primitive of $f$ ). In the above relation, the quantity $\frac{\delta \mathcal{F}}{\delta u}$ can be seen as a generalized force which arises as a consequence of the tendency of the free energy to decay towards a minimum. Thus, the relation $\frac{\partial u}{\partial t}=-\frac{\delta \mathcal{F}}{\delta u}$ means that the response of $u$ to the generalized force is instantaneous. Now, it has recently been proposed (see, e.g., [RDN]) that, in certain situations, the response of $u$ to the generalized force should be subject to a delay expressed by a time dependent relaxation kernel, i.e.,

$$
\frac{\partial u}{\partial t}=-\int_{-\infty}^{t} k(t-s) \frac{\delta \mathcal{F}}{\delta u}(s) d s,
$$

for a proper relaxation kernel $k$. A simple and classical choice of kernel reads

$$
k(t)=\frac{1}{\epsilon} e^{-\frac{t}{\epsilon}} .
$$

When $\epsilon \rightarrow 0$, then, $k(t) \rightarrow \delta_{0}$ (the Dirac mass at 0 ) and we recover $(0.1)_{2}$ for $\epsilon=0$. Now, when $\epsilon>0$, we find, by differentiation with respect to $t,(0.1)_{2}$.

In [GrP2] (see also [GrP1]), the authors studied the long time behavior of (0.1) with homogeneous Dirichlet boundary conditions for the temperature and homogeneous Neumann boundary conditions for the order parameter. We will consider such more physical boundary conditions, which generate additional difficulties such as, in particular, regularity problems, in a forthcoming article (these difficulties arise from the coupling and, if one deals with the hyperbolic equation only, Neumann boundary conditions can be handled provided that one adds and subtracts $u$ in equation $(0.1)_{2}$ and redefines $f(u)$ as $f(u)-u$ ). In particular, they proved the existence of the global attractor $\mathcal{A}_{\varepsilon}$, which is a compact, invariant by the flow set which attracts uniformly the bounded sets of initial data as time goes to infinity (see, e.g., [BV], [CV], $[\mathrm{H}]$, 
$[\mathrm{L}]$ and $[\mathrm{T}]$ for extensive reviews on this subject). Actually, for $\varepsilon=0$, one considers a proper lifting of the global attractor associated with the corresponding parabolic system; indeed, the phase spaces for $\varepsilon>0$ and $\varepsilon=0$ are not the same. Furthermore, the authors also proved an upper semicontinuity property of $\mathcal{A}_{\varepsilon}$ as $\varepsilon \rightarrow 0^{+}$. Finally, when $H$ has quadratic growth, they proved the existence of an exponential attractor $\mathcal{M}_{\varepsilon}$ (which is a compact, positively invariant set which contains the global attractor, has finite fractal dimension and attracts exponentially the bounded sets of initial data, see $[\mathrm{EFNT}]$ ), whose fractal dimension is uniformly (with respect to $\varepsilon \rightarrow 0^{+}$) bounded whenever $H$ is linear. As a consequence, one has a similar result for the global attractor $\mathcal{A}_{\varepsilon}$.

In [GrP1] and [GrP2], the authors only considered regular nonlinear functions $f$ (and, more precisely, functions $f$ with cubic growth). Now, more general nonlinearities (e.g., of logarithmic type), which requires the order parameter to belong to the physically relevant interval, for instance, $(-1,1)$, are also important from the thermodynamic point of view and they appear as natural generalization of polynomial nonlinearities (see, e.g., [BrS] and the references therein). The main difficulty, when considering such nonlinearities, is just ensuring that the order parameter does remain in $(-1,1)$ (of course, such a result would be also important to prove for regular nonlinearities; fortunately, even though this is not available in general, it is still possible to study the system in that case).

In [FGMZ] (see also [EfMZ2], [MZ1] and [MZ2]), the authors gave a general construction of robust (with respect to perturbations) exponential attractors, based on a recent construction of exponential attractors given in [EfMZ1] and valid in Banach spaces. We can note that this construction of robust exponential attractors is not based on the study of stationary solutions and their unstable manifolds, as it is the case for regular (and robust) global attractors (see [BV] and [T]) and should therefore apply to more situations: indeed, in general, global attractors are only upper semicontinuous with respect to perturbations.

Our aim in this article is to prove such a result for system (0.1) with a singular nonlinear term $f$. One of the main difficulties is to prove the existence of solutions when $\varepsilon>0$. When $\varepsilon=0$, in which case the system is parabolic, we can prove the existence of solutions by using some monotonicity arguments and the maximum principle (see Section 1). When $\varepsilon>0$, since the second equation of (0.1) is hyperbolic, we cannot use such arguments. However, we can use, in Section 2, some generalization of a perturbation technique developed in [Z] (for the study of nonlinear hyperbolic equations with supercritical nonlinearities) in order to prove that the solutions of (0.1) for $\varepsilon>0$ remain, in some sense, close to the corresponding solutions of the limit parabolic system when $\varepsilon$ is small and when the initial data are not too large. Furthermore, we are only able to prove the existence of strong solutions in that case. Then, in Section 3, we obtain several estimates on the difference of solutions of (0.1) and on the difference of solutions of (0.1) with $\varepsilon>0$ and $\varepsilon=0$ which are necessary to construct robust exponential attractors (in particular, we need the first term of the asymptotic expansion near $t=0$ as $\varepsilon \rightarrow 0^{+}$). Finally, in Section 4, 
we apply the abstract result of [FGMZ] to construct a continuous (as $\varepsilon \rightarrow 0^{+}$) family of exponential attractors for (0.1). Several useful uniform (as $\varepsilon \rightarrow 0^{+}$) estimates on linear hyperbolic problems are collected in an appendix.

Main assumptions. We assume that the functions $H:[-1,1] \rightarrow \mathbb{R}$ and $f$ : $(-1,1) \rightarrow \mathbb{R}$ satisfy the following assumptions:

$$
\left\{\begin{array}{l}
\text { 1. } f \in C^{3}(-1,1), \quad H \in C^{3}([-1,1]), \quad f(0)=0, \\
\text { 2. } \lim _{r \rightarrow \pm 1} f(r)= \pm \infty \\
\text { 3. } \lim _{r \rightarrow \pm 1} f^{\prime}(r)=+\infty
\end{array}\right.
$$

and that the external forces $g$ belong to the space $L^{\infty}(\Omega)$. We recall that a typical example of function $f$ is

$$
f(r)=-\kappa_{0} r+\kappa_{1} \ln \frac{1+r}{1-r}, \quad \forall r \in(-1,1),
$$

where $0<\kappa_{0}<\kappa_{1}$.

It is worth emphasizing that equations (0.1) have a sense only if

$$
-1<u(t, x)<1
$$

for almost all $(t, x) \in \mathbb{R}_{+} \times \Omega$. That is the reason why it is natural to introduce the following quantity:

$$
\mathbb{D}[u(t)]:=\left(1-\|u(t)\|_{L^{\infty}}\right)^{-1} .
$$

We also introduce, for every $s \geq 0$, the standard energy norm for the second equation of $(0.1)$ :

$$
\|(u, v)\|_{\mathcal{E}^{s}(\varepsilon)}^{2}:=\|u\|_{H^{s+1}}^{2}+\varepsilon\|v\|_{H^{s}}^{2}+\|v\|_{H^{s-1}}^{2}
$$

Thus, the space $\mathcal{E}^{s}(\varepsilon)$ coincides with $\left[H^{s+1}(\Omega) \times H^{s}(\Omega)\right] \cap\left\{\left.\xi\right|_{\partial \Omega}=0\right\}$ if $\varepsilon>0$ and with $\left[H^{s+1}(\Omega) \times H^{s-1}(\Omega)\right] \cap\left\{\left.\xi\right|_{\partial \Omega}=0\right\}$ if $\varepsilon=0$, whenever the traces make sense. For $s=0$, we will write $\mathcal{E}(\varepsilon)$ instead of $\mathcal{E}^{0}(\varepsilon)$.

\section{$\S 1$ The LIMIT PARABOLIC SYSTEM}

In this section, we consider the limit case $(\varepsilon=0)$ of system $(0.1)$ which reads

$$
\left\{\begin{array}{l}
\partial_{t}(\theta+H(u))-\Delta_{x} \theta=0,\left.\quad \theta\right|_{\partial \Omega}=0,\left.\quad \theta\right|_{t=0}=\theta_{0} \\
\partial_{t} u-\Delta_{x} u+f(u)-H^{\prime}(u) \theta=g,\left.\quad u\right|_{\partial \Omega}=0,\left.\quad u\right|_{t=0}=u_{0} .
\end{array}\right.
$$

We start with the following theorem. 
Theorem 1.1. Let the nonlinearities $H$ and $f$ satisfy assumptions (0.2) and $g$ belong to $L^{\infty}(\Omega)$. Then, for any initial data satisfying

$$
\mathbb{D}[u(0)]+\|\theta(0)\|_{H^{2}}^{2}+\|u(0)\|_{H^{2}}^{2}<\infty
$$

equation (1.1) possesses a unique solution $(\theta(t), u(t))$ which satisfies the estimate

$$
\begin{aligned}
\mathbb{D}[u(t)]+\|\theta(t)\|_{H^{2}}^{2} & +\|u(t)\|_{H^{2}}^{2} \leq \\
& \leq Q\left(\mathbb{D}[u(0)]+\|\theta(0)\|_{H^{2}}^{2}+\|u(0)\|_{H^{2}}^{2}\right) e^{-\alpha t}+Q\left(\|g\|_{L^{\infty}}\right)
\end{aligned}
$$

where the positive constant $\alpha$ and the monotonic function $Q$ are independent of $(\theta(0), u(0))$.

Proof. We first derive the a priori estimate (1.3), assuming that $\left(\theta(t), \xi_{u}(t)\right)$ is a sufficiently regular solution of (1.1) which is separated from the singular points of $f$, i.e., $\|u\|_{L^{\infty}\left(\mathbb{R}_{+} \times \Omega\right)}<1$. To this end, we need the following Lemmata.

Lemma 1.1. Let the above assumptions hold. Then, the following estimate is valid

$$
\begin{aligned}
& \|\theta(t)\|_{H^{1}}^{2}+\|u(t)\|_{H^{1}}^{2}+ \\
& +\int_{0}^{t} e^{-\alpha(t-s)}\left(\left\|\partial_{t} \theta(s)\right\|_{L^{2}}^{2}+\|\theta(s)\|_{H^{2}}^{2}+\left\|\partial_{t} u(s)\right\|_{L^{2}}^{2}\right) d s \leq \\
& \quad \leq Q\left(\mathbb{D}[u(0)]+\|\theta(0)\|_{H^{1}}^{2}+\|u(0)\|_{H^{1}}^{2}\right) e^{-\alpha t}+Q\left(\|g\|_{L^{2}}\right),
\end{aligned}
$$

for appropriate positive constant $\alpha$ and monotonic function $Q$.

Proof of Lemma 1.1. Multiplying the first equation of (1.1) by $\theta(t)$ and the second one by $\partial_{t} u(t)$ and integrating over $\Omega$, we have

$$
\begin{aligned}
\partial_{t}\left(\|\theta(t)\|_{L^{2}}^{2}+\left\|\nabla_{x} u(t)\right\|_{L^{2}}^{2}\right. & \left.+2(F(u(t)), 1)_{L^{2}}\right)+ \\
& +2\left\|\partial_{t} u(t)\right\|_{L^{2}}^{2}+2\left\|\nabla_{x} \theta(t)\right\|_{L^{2}}^{2}=2\left(g, \partial_{t} u(t)\right)_{L^{2}},
\end{aligned}
$$

where $F(r):=\int_{0}^{r} f(s) d s$ and $(u, v)_{L^{2}}$ denotes the standard inner product in $L^{2}(\Omega)$. Multiplying the second equation of (1.1) by $\alpha u(t)$, where $\alpha>0$ is small enough, and integrating over $\Omega$, we deduce that

$$
\begin{aligned}
\alpha \partial_{t}\|u(t)\|_{L^{2}}^{2}+2 \alpha\left\|\nabla_{x} u(t)\right\|_{L^{2}}^{2}+ & 2 \alpha(f(u(t)), u(t))_{L^{2}-} \\
& -2 \alpha\left(H^{\prime}(u(t)) \theta(t), u(t)\right)_{L^{2}}=2 \alpha(g, u(t))_{L^{2}} .
\end{aligned}
$$

Summing equations (1.5) and (1.6) and using the fact that, a priori, $\|u(t)\|_{L^{\infty}} \leq 1$ and the obvious inequalities

$$
-C \leq F(r) \leq \underset{5}{f(r) r+C, \quad \forall r \in(-1,1),}
$$


we obtain

$$
\begin{aligned}
& \partial_{t}\left[\|\theta(t)\|_{L^{2}}^{2}+\left\|\nabla_{x} u(t)\right\|_{L^{2}}^{2}+\alpha\|u(t)\|_{L^{2}}^{2}+2(F(u(t)), 1)_{L^{2}}\right]+ \\
& \quad+\alpha\left[\|\theta(t)\|_{L^{2}}^{2}+\left\|\nabla_{x} u(t)\right\|_{L^{2}}^{2}+\alpha\|u(t)\|_{L^{2}}^{2}+2(F(u(t)), 1)_{L^{2}}\right]+ \\
& \quad+\left\|\partial_{t} u(t)\right\|_{L^{2}}^{2}+2 \alpha\left\|\nabla_{x} \theta(t)\right\|_{L^{2}}^{2} \leq C\left(1+\|g\|_{L^{2}}^{2}\right),
\end{aligned}
$$

where $\alpha>0$ is small enough and $C$ is independent of the solution $(\theta, u)$. Applying Gronwall's inequality to (1.8), we obtain the $u$-part of estimate (1.4). In order to have the $\theta$-part, it is sufficient to rewrite the first equation of (1.1) as follows:

$$
\partial_{t} \theta-\Delta_{x} \theta=h_{u}(t):=-H^{\prime}(u(t)) \partial_{t} u(t),
$$

apply the following classical $L^{2}$-regularity estimate for the heat equation to (1.9):

$$
\begin{gathered}
\|\theta(t)\|_{H^{1}}^{2}+\int_{0}^{t} e^{-\alpha(t-s)}\left(\left\|\partial_{t} \theta(s)\right\|_{L^{2}}^{2}+\|\theta(s)\|_{H^{2}}^{2}\right) d s \leq \\
\leq C\|\theta(0)\|_{H^{1}}^{2} e^{-\alpha t}+C \int_{0}^{t} e^{-\alpha(t-s)}\left\|h_{u}(s)\right\|_{L^{2}}^{2} d s
\end{gathered}
$$

and use the estimate for the integral of $\left\|\partial_{t} u(t)\right\|_{L^{2}}^{2}$ obtained above in order to estimate the right-hand side of the last inequality. This finishes the proof of Lemma 1.1 .

Our purpose is now to obtain estimates for the $H^{2}$-norms of $\theta(t)$ and $u(t)$.

Lemma 1.2. Let the above assumptions hold. Then, the following estimate is valid

$$
\begin{aligned}
\|\theta(t)\|_{H^{2}}+\|u(t)\|_{H^{2}} \leq Q\left(\mathbb{D}[u(0)]+\|\theta(0)\|_{H^{2}}+\|u(0)\|_{H^{2}}\right) e^{-\alpha t}+ & \\
& +Q\left(\|g\|_{L^{\infty}}\right),
\end{aligned}
$$

for some positive constant $\alpha$ and monotonic function $Q$.

Proof of Lemma 1.2. We differentiate the second equation of (1.1) with respect to $t$ and set $p(t):=\partial_{t} u(t)$. Then, we have

$$
\begin{aligned}
\partial_{t} p-\Delta_{x} p+f^{\prime}(u(t)) p=H^{\prime}(u(t)) \partial_{t} \theta(t) & +H^{\prime \prime}(u(t)) \partial_{t} u(t) \theta(t) \\
& \left.p\right|_{t=0}=\Delta_{x} u_{0}-f\left(u_{0}\right)+H^{\prime}\left(u_{0}\right) \theta_{0}+g .
\end{aligned}
$$

Multiplying this equation by $p(t)$, integrating over $\Omega$ and using the facts that $f^{\prime}(r) \geq$ $-K$ and $\|u(t)\|_{L^{\infty}} \leq 1$, we infer

$$
\begin{gathered}
\frac{1}{2} \frac{d}{d t}\|p(t)\|_{L^{2}}^{2}+\left\|\nabla_{x} p(t)\right\|_{L^{2}}^{2} \leq(K+1)\left\|\partial_{t} u(t)\right\|_{L^{2}}^{2}+ \\
6{ }^{+C\left\|\partial_{t} \theta(t)\right\|_{L^{2}}^{2}+C_{1}\left(|\theta(t)|,|p(t)|^{2}\right)_{L^{2}}}
\end{gathered}
$$


Estimating the last term in the right-hand side of (1.12) by Hölder's inequality with exponents 4 and $4 / 3$ and using the interpolation inequality $\|\cdot\|_{L^{4}} \leq C\|\cdot\|_{L^{2}}^{1 / 4}\|\cdot\|_{H^{1}}^{3 / 4}$, we have

$$
\begin{aligned}
C_{1}\left(|\theta(t)|,|p(t)|^{2}\right)_{L^{2}} \leq C_{2}\|\theta(t)\|_{L^{2}}\left\|\partial_{t} u(t)\right\|_{L^{2}}^{1 / 2}\left\|\nabla_{x} p(t)\right\|_{L^{2}}^{3 / 2} & \leq \\
& \leq C_{3}\|\theta(t)\|_{L^{2}}^{4}\left\|\partial_{t} u(t)\right\|_{L^{2}}^{2}+\frac{1}{4}\left\|\nabla_{x} p(t)\right\|_{L^{2}}^{2} .
\end{aligned}
$$

We also note that, obviously,

$$
\begin{aligned}
\|p(0)\|_{L^{2}} \leq\left\|u_{0}\right\|_{H^{2}}+\left\|f\left(u_{0}\right)\right\|_{L^{2}}+\|g\|_{L^{2}}+C\left\|\theta_{0}\right\|_{L^{2}} \leq \\
\leq Q\left(\mathbb{D}[u(0)]+\left\|u_{0}\right\|_{H^{2}}+\left\|\theta_{0}\right\|_{L^{2}}\right)+\|g\|_{L^{2}},
\end{aligned}
$$

for an appropriate monotonic increasing function $Q$. Consequently, applying Gronwall's inequality to (1.12) and using (1.13) and (1.4), we deduce that

$$
\begin{aligned}
\left\|\partial_{t} u(t)\right\|_{L^{2}}^{2}+\int_{0}^{t} e^{-\alpha(t-s)}\left\|\partial_{t} u(s)\right\|_{H^{1}}^{2} d s \leq \\
\quad \leq Q\left(\mathbb{D}[u(0)]+\|\theta(0)\|_{H^{1}}+\|u(0)\|_{H^{2}}\right) e^{-\alpha t}+Q\left(\|g\|_{L^{\infty}}\right),
\end{aligned}
$$

for appropriate positive constant $\alpha$ and monotonic function $Q$.

We now multiply the second equation of (1.1) by $\Delta_{x} u(t)$, integrate over $\Omega$ and use the fact that $f^{\prime}(r) \geq-K$. Then, we have

$$
\left\|\Delta_{x} u(t)\right\|_{L^{2}}^{2} \leq 2 K\left\|\nabla_{x} u(t)\right\|_{L^{2}}^{2}+2\|g\|_{L^{2}}^{2}+C\|\theta(t)\|_{L^{2}}^{2}+2\left\|\partial_{t} u(t)\right\|_{L^{2}}^{2} .
$$

Estimating the right-hand side of (1.15) by (1.14) and (1.4), we infer

$$
\|u(t)\|_{H^{2}}^{2} \leq Q\left(\mathbb{D}[u(0)]+\|\theta(0)\|_{H^{1}}+\|u(0)\|_{H^{2}}\right) e^{-\alpha t}+Q\left(\|g\|_{L^{\infty}}\right),
$$

for appropriate positive constant $\alpha$ and monotonic function $Q$. Thus, the estimate of the $H^{2}$-norm of $u(t)$ is obtained. In order to obtain an analogous estimate for $\theta(t)$, we rewrite the first equation of (1.1) in the form (1.9) and note that, due to Hölder's inequality and the embedding $H^{1} \subset L^{6}$,

$$
\begin{aligned}
\left\|h_{u}(t)\right\|_{H^{1}}^{2} \leq C\left\|\partial_{t} u(t)\right\|_{H^{1}}^{2}+C\left\|\partial_{t} u(t) \nabla_{x} u(t)\right\|_{L^{2}}^{2} & \leq \\
& \leq C_{1}\left(1+\|u(t)\|_{H^{2}}^{2}\right)\left\|\partial_{t} u(t)\right\|_{H^{1}}^{2} .
\end{aligned}
$$

We also recall that $\left.h_{u}(t)\right|_{\partial \Omega}=0$. Then, applying the $H^{2}$-regularity theorem to the heat equation (1.9) and using (1.17), (1.16) and (1.14), we have

$$
\begin{gathered}
\|\theta(t)\|_{H^{2}}^{2} \leq C\|\theta(0)\|_{H^{2}}^{2} e^{-\alpha t}+C \int_{0}^{t} e^{-\alpha(t-\tau)}\left\|h_{u}(\tau)\right\|_{H^{1}}^{2} d \tau \leq \\
\leq C\|\theta(0)\|_{H^{2}}^{2}+C_{1} \sup _{\tau \in[0, t]}\left\{e^{-\alpha(t-\tau)}\left(1+\|u(\tau)\|_{H^{2}}^{2}\right)^{2}\right\}+ \\
+C_{1}\left(\int_{0}^{t} e^{-\alpha(t-\tau)}\left\|\partial_{t} u(\tau)\right\|_{H^{1}}^{2} d \tau\right)^{2} \leq \\
\leq Q\left(\mathbb{D}[u(0)]+\|\theta(0)\|_{H^{2}}+\|u(0)\|_{H^{2}}\right) e^{-\alpha t}+Q\left(\|g\|_{L^{\infty}}\right), \\
7
\end{gathered}
$$


for appropriate positive constant $\alpha$ and monotonic function $Q$. Estimate (1.18) finishes the proof of Lemma 1.2.

We are now ready to finish the proof of Theorem 1.1. To this end, we only need to estimate $\mathbb{D}[u(t)]$. In order to do so, we rewrite the second equation of (1.1) as follows:

$$
\partial_{t} u-\Delta_{x} u+f(u)=h_{\theta, u}(t):=g+H^{\prime}(u(t)) \theta(t),
$$

and note that, due to the embedding $H^{2} \subset C(\bar{\Omega})$ and Lemma 1.2, we have

$$
\left\|h_{\theta, u}(t)\right\|_{L^{\infty}} \leq Q\left(\mathbb{D}[u(0)]+\|\theta(0)\|_{H^{2}}+\|u(0)\|_{H^{2}}\right) e^{-\alpha t}+Q\left(\|g\|_{L^{\infty}}\right),
$$

for some positive constant $\alpha$ and monotonic function $Q$. Thus, due to the comparison principle for second order parabolic PDEs, we deduce the following inequalities:

$$
y_{-}(t) \leq u(t, x) \leq y_{+}(t), \quad(t, x) \in \mathbb{R}_{+} \times \Omega,
$$

where the functions $y_{ \pm}(t)$ solve the following ODEs:

$$
y_{ \pm}^{\prime}(t)+f\left(y_{ \pm}(t)\right)=h_{ \pm}(t):= \pm\left\|h_{\theta, u}(t)\right\|_{L^{\infty}}, \quad y_{ \pm}(0):= \pm\|u(0)\|_{L^{\infty}} .
$$

Moreover, it is not difficult to verify, using assumptions (0.2) on the nonlinearity $f$ (see, e.g., [MZ2, Appendix]), that the solutions of (1.22) satisfy the following inequalities:

$$
\left\{\begin{array}{l}
\left\|y_{ \pm}(t)\right\|_{L^{\infty}} \leq 1-\delta\left(\mathbb{D}[u(0)]+\left\|h_{ \pm}\right\|_{L^{\infty}([0,1])}\right), \quad 0 \leq t \leq 1, \\
\left\|y_{ \pm}(t+1)\right\|_{L^{\infty}} \leq 1-\delta\left(\left\|h_{ \pm}\right\|_{L^{\infty}([t, t+1])}\right), \quad t \geq 0,
\end{array}\right.
$$

where the strictly positive and monotonic decreasing function $\delta$ depends on $f$, but it is independent of $t$ and $y_{ \pm}(t)$. Estimates (1.20), (1.21) and (1.23) imply that

$$
\mathbb{D}[u(t)] \leq Q\left(\mathbb{D}[u(0)]+\|\theta(0)\|_{H^{2}}+\|u(0)\|_{H^{2}}\right) e^{-\alpha t}+Q\left(\|g\|_{L^{\infty}}\right),
$$

for appropriate positive constant $\alpha$ and monotonic increasing function $Q$. This, together with estimate (1.10), finish the proof of the a priori estimate (1.3). In particular, estimate (1.3) implies that

$$
\|u(t)\|_{L^{\infty}} \leq 1-\delta, \quad \forall t \geq 0
$$

for some positive constant $\delta$ and, consequently, every solution $(\theta(t), u(t))$ of $(1.1)$ is a priori strictly separated from the singular points $r= \pm 1$ of the nonlinearity $f$. Thus, the existence and uniqueness of a solution $(\theta(t), u(t))$ of problem (1.1) can be now verified exactly as in the case of regular nonlinearities, see, e.g., [GrP1] or [GrP2], and Theorem 1.1 is proven.

The following theorem gives the smoothing property for system (1.1). 
Theorem 1.2. Let the nonlinearities $H$ and $f$ satisfy assumptions (0.2). Then, any solution $(\theta(t), u(t))$ satisfies the following estimate

$$
\mathbb{D}[u(t)]+\|\theta(t)\|_{H^{2}}+\|u(t)\|_{H^{2}} \leq Q\left(t^{-1}+\|\theta(0)\|_{L^{2}}\right) e^{-\alpha t}+Q\left(\|g\|_{L^{\infty}}\right), \quad t>0
$$

where the positive constant $C$ and the monotonic function $Q$ are independent of the solution $(\theta, u)$.

Proof. We first note that, due to estimate (1.3), it is sufficient to verify (1.24) for $t \leq 1$ only. We divide the proof of estimate (1.24) with $t \leq 1$ into several lemmata.

Lemma 1.3. Let the above assumptions hold. Then, the following estimate is valid for all $0 \leq t \leq 1$

$$
\begin{array}{r}
\|\theta(t)\|_{L^{2}}^{2}+\int_{0}^{t}\left(\|\theta(s)\|_{H^{1}}^{2}+\|u(s)\|_{H^{1}}^{2}+(|f(u(s))|,|u(s)|)_{L^{2}}\right) d s \leq \\
\leq C\left(\|\theta(0)\|_{L^{2}}^{2}+1\right),
\end{array}
$$

where the constant $C$ is independent of the solution $(\theta(t), u(t))$.

Proof of Lemma 1.3. Integrating the first equation of (1.1) over $[0, t]$, we have

$$
\theta(t)-\Delta_{x} \int_{0}^{t} \theta(s) d s=\theta(0)+H(u(0))-H(u(t)) .
$$

Multiplying this equation by $\theta(t)$, integrating over $[0, t] \times \Omega$ and using the fact that $\|H(u(t))\|_{L^{\infty}} \leq\|H\|_{C([-1,1])}:=L$, we have

$$
\int_{0}^{t}\|\theta(s)\|_{L^{2}}^{2} d s+\left\|\nabla_{x}\left(\int_{0}^{t} \theta(s) d s\right)\right\|_{L^{2}}^{2} \leq C\left(\|\theta(0)\|_{L^{2}}^{2}+1\right),
$$

where the constant $C$ depends on $L$, but it is independent of $t \in[0,1]$ and $\theta(t)$. Multiplying now the second equation of $(1.1)$ by $u(t)$, integrating over $[0, t] \times \Omega$ and using (1.26) and the fact that $\|u(t)\|_{L^{\infty}} \leq 1$, we obtain

$$
\int_{0}^{t}\left(\|u(s)\|_{H^{1}}^{2}+(|f(u(s))|,|u(s)|)_{L^{2}}\right) d s \leq C_{1}\left(\|\theta(0)\|_{L^{2}}^{2}+1+\|g\|_{L^{2}}^{2}\right),
$$

where the constant $C_{1}$ is independent of the solution $(\theta, u)$ (here, we also implicitly used the fact that, due to $\left.(0.2)_{2}, f(r) r \geq-C\right)$. Thus, it only remains to obtain the $\theta$-part of estimate (1.25). To this end, we transform the first equation of (1.1) as follows:

$$
\partial_{t}(\theta+H(u))-\Delta_{x}(\theta+H(u))=-\Delta_{x} H(u),
$$


multiply (1.28) by $\theta(t)+H(u(t))$ and integrate over $[0, t] \times \Omega$. Then, after standard transformations, we have

$$
\begin{aligned}
& \| \theta(t)+ H(u(t))\left\|_{L^{2}}^{2}+\int_{0}^{t}\right\| \theta(s)+H(u(s)) \|_{H^{1}}^{2} d s \leq \\
& \leq C_{2}\left(\|\theta(0)+H(u(0))\|_{L^{2}}^{2}+\int_{0}^{t}\left\|\nabla_{x} H(u(s))\right\|_{L^{2}}^{2} d s\right) \leq \\
& \leq C_{3}\left(1+\|\theta(0)\|_{L^{2}}^{2}+\int_{0}^{t}\left\|\nabla_{x} u(s)\right\|_{L^{2}}^{2} d s\right) .
\end{aligned}
$$

Using now estimate (1.27) in order to estimate the last term in the right-hand side of (1.29), we deduce the desired estimate for $\theta(t)$ and finish the proof of Lemma 1.3.

Lemma 1.4. Let the above assumptions hold. Then, the following estimate is valid

$$
\begin{aligned}
& t\left(\|\theta(t)\|_{H^{1}}^{2}+\|u(t)\|_{H^{1}}^{2}+\int_{\Omega} F(u(t)) d x\right)+ \\
& \quad+\int_{0}^{t} s\left(\left\|\partial_{t} \theta(s)\right\|_{L^{2}}^{2}+\left\|\partial_{t} u(s)\right\|_{L^{2}}^{2}\right) d s \leq C\left(\|\theta(0)\|_{L^{2}}^{2}+\|g\|_{L^{2}}^{2}+1\right)
\end{aligned}
$$

where $F(r):=\int_{0}^{r} f(s) d s$ and the constant $C$ is independent of $t \in[0,1]$ and of the solution $(\theta, u)$.

Proof of Lemma 1.4. Multiplying the second equation of (1.1) by $t \partial_{t} u(t)$ and integrating over $[0, t] \times \Omega$, we obtain, after simple transformations

$$
\begin{aligned}
& t\left(\|u(t)\|_{H^{1}}^{2}+\int_{\Omega} F(u(t)) d x\right)+\int_{0}^{t} s\left\|\partial_{t} u(s)\right\|_{L^{2}}^{2} d s \leq \\
& \leq C \int_{0}^{t}\left(\|F(u(s))\|_{L^{1}}+\|u(s)\|_{H^{1}}^{2}+1+\|\theta(s)\|_{L^{2}}^{2}\right) d s
\end{aligned}
$$

Inserting estimates (1.25) into the right-hand side of (1.31) and using estimates (1.7), we deduce the $u$-part of estimate (1.30). The $\theta$-part of this estimate can be now obtained in a standard way by multiplying the first equation of (1.1) by $t \partial_{t} \theta(t)$ and integrating over $[0, t] \times \Omega$. This finishes the proof of Lemma 1.4.

Lemma 1.5. Let the above assumptions hold. Then, the following estimate is valid

$$
t^{2}\left(\left\|\partial_{t} u(t)\right\|_{L^{2}}^{2}+\|\theta(t)\|_{L^{\infty}}^{2}\right) \leq C\left(1+\|\theta(0)\|_{L^{2}}^{6}+\|g\|_{L^{2}}^{6}\right),
$$

where the constant $C$ is independent of the solution $(\theta, u)$.

Proof of Lemma 1.5. We differentiate the second equation of (1.1) with respect to $t$ and set $p(t):=\partial_{t} u(t)$. This function satisfies the equation

$$
\begin{gathered}
\partial_{t} p-\Delta_{x} p+f^{\prime}(u(t)) p-H^{\prime \prime}(u(t)) \theta(t) p=H^{\prime}(u(t)) \partial_{t} \theta(t) . \\
10
\end{gathered}
$$


We multiply this equation by $t^{2} p(t)$ and integrate over $[0, t] \times \Omega$. Then, using estimate (1.13) and the facts that $t \leq 1$ and $f^{\prime}(r) \geq-K$, we infer

$$
\begin{aligned}
t^{2}\|p(t)\|_{L^{2}}^{2}+ & \int_{0}^{t} s^{2}\|p(s)\|_{H^{1}}^{2} d s \leq \\
\leq & C \int_{0}^{t} s\left(\left\|\partial_{t} u(s)\right\|_{L^{2}}^{2}+\left\|\partial_{t} \theta(s)\right\|_{L^{2}}^{2}\right) d s+ \\
& \quad+\frac{1}{4} \int_{0}^{t} s^{2}\|p(s)\|_{H^{1}}^{2} d s+C \int_{0}^{t} s^{2}\|\theta(s)\|_{H^{1}}^{4}\left\|\partial_{t} u(s)\right\|_{L^{2}}^{2} d s .
\end{aligned}
$$

Estimating the right-hand side of (1.34) by (1.30), we finally have

$$
t^{2}\left\|\partial_{t} u(t)\right\|_{L^{2}}^{2} \leq C_{4}\left(1+\|\theta(0)\|_{L^{2}}^{6}+\|g\|_{L^{2}}^{6}\right) .
$$

Thus, the $u$-part of (1.32) is proven. In order to obtain the $\theta$-part, we rewrite the first equation of (1.1) as follows:

$$
\partial_{t}(t \theta(t))+\Delta_{x}(t \theta(t))=h_{u}(t):=t H^{\prime}(u(t)) \partial_{t} u(t)+\theta(t),\left.\quad(t \theta(t))\right|_{t=0}=0,
$$

where, due to (1.35), we have $\left\|h_{u}(t)\right\|_{L^{2}} \leq C\left(1+\|\theta(0)\|_{L^{2}}^{2}+\|g\|_{L^{2}}^{2}\right)$. Applying now the classical $L^{\infty}$-estimate (see, e.g., [LSU]) to the heat equation that we obtain and using (1.25) in order to estimate the $L^{2}$-norm of $\theta(t)$, we deduce that

$$
t\|\theta(t)\|_{L^{\infty}} \leq C\left\|h_{u}\right\|_{L^{\infty}\left([0,1], L^{2}(\Omega)\right)} \leq C_{5}\left(1+\|\theta(0)\|_{L^{2}}^{2}+\|g\|_{L^{2}}^{2}\right)
$$

and Lemma 1.5 is proven.

Lemma 1.6. Let the above assumptions hold. Then, the following estimate is valid

$$
\mathbb{D}[u(t)] \leq Q\left(t^{-1}+\|\theta(0)\|_{L^{2}}\right)+Q\left(\|g\|_{L^{\infty}}\right), t \in(0,1]
$$

for an appropriate monotonic function $Q$ which is independent of $t \in[0,1]$ and of the solution $(\theta, u)$.

Proof of Lemma 1.6. We rewrite the second equation of (1.1) in the form (1.19). Then, due to Lemma 1.5, we have, instead of (1.20)

$$
t\left\|h_{\theta, u}(t)\right\|_{L^{\infty}} \leq C\left(1+\|\theta(0)\|_{L^{2}}^{2}+\|g\|_{L^{\infty}}^{2}\right) .
$$

Moreover, thanks to the comparison principle, we again have inequalities (1.21) for the function $u(t, x)$, where the functions $y_{ \pm}(t)$ solve the ODEs (1.22), and, analogously to (1.23), it is not difficult to verify that the solutions of (1.22) are well defined and satisfy the following improved version of the "smoothing property" $(1.23)_{2}$ :

$$
\left|y_{ \pm}(t)\right| \leq 1-\delta\left(t^{-1}+\left\|h_{ \pm}\right\|_{L^{\infty}([t / 2,1])}\right), \quad t \in(0,1]
$$


where the positive and monotonic decreasing function $\delta$ (which satisfies $\lim _{z \rightarrow \infty} \delta(z)$ $=0$ ) depends only on $f$ and it is independent of $y_{ \pm}(0)$ (see, e.g., [MZ2, Appendix] for details). Estimates (1.39), (1.40) and (1.21) give (1.38) and Lemma 1.6 is proven.

Estimate (1.38) shows that the solution $(\theta, u)$ is strictly separated from the singular points of the nonlinearity $f$ for every $t>0$ and, therefore, the $H^{2}$-smoothing part of estimate (1.24) can be now derived exactly as in the case of regular nonlinearities. This finishes the proof of Theorem 1.2.

We now obtain the Hölder-Lipschitz continuity of the solutions of problem (1.1).

Theorem 1.3. Let the assumptions of Theorem 1.1 be satisfied and let $\left(\theta_{1}(t), u_{1}(t)\right)$ and $\left(\theta_{2}(t), u_{2}(t)\right)$ be two solutions of problem $(1.1)$ such that $\mathbb{D}\left[u_{i}(t)\right]<\infty$ for $i=1,2$ and $t \in \mathbb{R}_{+}$. Then, there exists a positive constant $\delta>0$ such that the following estimate is valid

$$
\begin{aligned}
\left\|\theta_{1}(t)-\theta_{2}(t)\right\|_{L^{2}}^{\delta}+\| u_{1}(t) & -u_{2}(t) \|_{L^{2}} \leq \\
\leq & C e^{K t}\left(\left\|\theta_{1}(0)-\theta_{2}(0)\right\|_{L^{2}}+\left\|u_{1}(0)-u_{2}(0)\right\|_{L^{2}}\right)
\end{aligned}
$$

where the constants $C$ and $K$ depend only on $f$ and $H$ and on the $L^{2}$-norms of the initial data $\theta_{1}(0)$ and $\theta_{2}(0)$.

Proof. We set $\bar{\theta}(t):=\theta_{1}(t)-\theta_{2}(t)$ and $\bar{u}(t):=u_{1}(t)-u_{2}(t)$. Then, these functions satisfy the following equations:

$$
\left\{\begin{array}{l}
\partial_{t} \bar{\theta}-\Delta_{x} \bar{\theta}=-\partial_{t}\left[H\left(u_{1}(t)\right)-H\left(u_{2}(t)\right)\right], \\
\partial_{t} \bar{u}-\Delta_{x} \bar{u}+l(t) \bar{u}=\left[H^{\prime}\left(u_{1}(t)\right) \theta_{1}(t)-H^{\prime}\left(u_{2}(t)\right) \theta_{2}(t)\right],
\end{array}\right.
$$

where $l(t):=\int_{0}^{1} f^{\prime}\left(s u_{1}(t)+(1-s) u_{2}(t)\right) d s$. In order to handle the first equation of (1.42), we need the following lemma.

Lemma 1.7. Let the function $v(t)$ solve the following equation:

$$
\partial_{t} v-\Delta_{x} v=\partial_{t} h(t),\left.\quad v\right|_{t=0}=v_{0},\left.\quad v\right|_{\partial \Omega}=0,
$$

for some $h \in H_{\text {loc }}^{1}\left(\mathbb{R}_{+}, L^{2}(\Omega)\right)$. Then, for all sufficiently small $\alpha \geq 0$, the following estimate is valid

$$
\begin{aligned}
\int_{0}^{t} e^{-\alpha(t-s)}\|v(s)\|_{L^{2}}^{2} d s \leq C\left(\|v(0)\|_{L^{2}}^{2}+\|h(0)\|_{L^{2}}^{2}\right) e^{-\alpha t}+ & \\
& +C \int_{0}^{t} e^{-\alpha(t-s)}\|h(s)\|_{L^{2}}^{2} d s
\end{aligned}
$$

where the constant $C$ is independent of $t$ and $\alpha$. 
Proof of Lemma 1.7. We set $w(t):=\int_{0}^{t} e^{-\beta(t-s)} v(s) d s$, for some $\beta \geq 0$. Then, this function satisfies the equation

$$
\begin{aligned}
\partial_{t} w(t)-\Delta_{x} w(t)=\tilde{h}(t):=e^{-\beta t}(v(0) & -h(0))+h(t)- \\
& -\beta \int_{0}^{t} e^{-\beta(t-s)} h(s) d s,\left.w\right|_{t=0}=0 .
\end{aligned}
$$

Applying the classical $L^{2}$-regularity estimate to the heat equation (1.45) and taking into account the fact that the first eigenvalue of $-\Delta_{x}$ with Dirichlet boundary conditions in $\Omega$ is strictly positive, we have

$$
\int_{0}^{t} e^{-\alpha(t-s)}\left(\left\|\partial_{t} w(s)\right\|_{L^{2}}^{2}+\left\|\Delta_{x} w(s)\right\|_{L^{2}}^{2}\right) d s \leq C \int_{0}^{t} e^{-\alpha(t-s)}\|\tilde{h}(s)\|_{L^{2}}^{2} d s,
$$

where $\alpha \geq 0$ is small enough, see, e.g., [LSU]. Noting that $v(t)=\partial_{t} w(t)+\beta w(t)$, fixing $\beta>\alpha$ and inserting the explicit expression for $\tilde{h}(t)$ given in (1.45) into the right-hand side of the previous estimate, we obtain estimate (1.44) and finish the proof of Lemma 1.7.

Applying Lemma 1.7 with $\alpha=0$ to the first equation of (1.42), we have

$$
\int_{0}^{t}\|\bar{\theta}(s)\|_{L^{2}}^{2} d s \leq C \int_{0}^{t}\|\bar{u}(s)\|_{L^{2}}^{2} d s+C\left(\|\bar{\theta}(0)\|_{L^{2}}^{2}+\|\bar{u}(0)\|_{L^{2}}^{2}\right),
$$

where the constant $C$ is independent of $t \geq 0$.

Using now Hölder's inequality, an appropriate interpolation inequality and the fact that, a priori, $\left\|u_{i}(t)\right\|_{L^{\infty}} \leq 1$, we have

$$
\begin{aligned}
& \left|\left(H^{\prime}\left(u_{1}\right) \theta_{1}-H^{\prime}\left(u_{2}\right) \theta_{2}, \bar{u}\right)_{L^{2}}\right| \leq C\|\bar{\theta}\|_{L^{2}}\|\bar{u}\|_{L^{2}}+ \\
& +C\left\|\theta_{1}\right\|_{L^{2}}\|\bar{u}\|_{L^{3}}\|\bar{u}\|_{L^{6}} \leq \\
& \quad \leq C_{1}\left(1+\left\|\theta_{1}\right\|_{L^{2}}^{4}\right)\|\bar{u}\|_{L^{2}}^{2}+1 / 2\|\bar{u}\|_{H^{1}}^{2}+C_{1}\|\bar{\theta}\|_{L^{2}}^{2},
\end{aligned}
$$

where the constant $C_{1}$ is independent of the solutions $\left(\theta_{1}, u_{1}\right)$ and $\left(\theta_{2}, u_{2}\right)$. Multiplying now the second equation of (1.42) by $\bar{u}(t)$, integrating over $\Omega$ and using estimate (1.47) and the fact that $l(t) \geq-K$ (due to $(0.2)$ ), we have

$$
\frac{d}{d t}\|\bar{u}(t)\|_{L^{2}}^{2}+\|\bar{u}(t)\|_{H^{1}}^{2} \leq C_{2}\left(1+\left\|\theta_{1}(t)\right\|_{L^{2}}^{4}\right)\|\bar{u}(t)\|_{L^{2}}^{2}+C_{2}\|\bar{\theta}(t)\|_{L^{2}}^{2} .
$$

Integrating inequality (1.48) over $[0, t]$ and using estimates (1.25) and (1.46), we deduce that

$$
\begin{aligned}
\|\bar{u}(t)\|_{L^{2}}^{2}+\int_{0}^{t}\|\bar{u}(s)\|_{H^{1}}^{2} d s \leq & C_{3}\left(\|\bar{u}(0)\|_{L^{2}}^{2}+\|\bar{\theta}(0)\|_{L^{2}}^{2}\right)+ \\
& +C_{3}\left(1+\left\|\theta_{1}(0)\right\|_{L^{2}}^{4}+\|g\|_{L^{2}}^{4}\right) \int_{0}^{t}\|\bar{u}(s)\|_{L^{2}}^{2} d s .
\end{aligned}
$$


Applying Gronwall's inequality to (1.49), we finally deduce that

$$
\|\bar{u}(t)\|_{L^{2}}^{2}+\int_{0}^{t}\|\bar{u}(s)\|_{H^{1}}^{2} d s \leq C e^{K t}\left(\|\bar{u}(0)\|_{L^{2}}^{2}+\|\bar{\theta}(0)\|_{L^{2}}^{2}\right),
$$

where the constants $C$ and $K$ only depend on the $L^{2}$-norm of $\theta_{1}(0)$. Thus, the $u$-part of estimate (1.41) is proven. In order to obtain the $\theta$-part, we need one more lemma.

Lemma 1.8. Let $v(t)$ solve the following equation

$$
\partial_{t} v-\Delta_{x} v=-\Delta_{x} \xi(t),\left.\quad v\right|_{t=0}=v_{0}
$$

where $v_{0} \in L^{2}(\Omega)$ and $\xi \in L^{2 /(1-\kappa)}\left([0, T], H^{1-\kappa}(\Omega)\right)$ for some $0<\kappa<1 / 2$. We also assume that $\left.\xi(t)\right|_{\partial \Omega} \equiv 0$. Then, the following estimate holds

$$
\|v(t)\|_{L^{2}} \leq C\left\|v_{0}\right\|_{L^{2}} e^{-\alpha t}+C\left(\int_{0}^{t} e^{-\alpha(t-s)}\|\xi(s)\|_{H^{1-\kappa}}^{2 /(1-\kappa)} d s\right)^{(1-\kappa) / 2}
$$

where the positive constants $C$ and $\alpha$ depend on $\kappa$, but they are independent of $\xi, t$ and $v$.

Proof. We first note that, without loss of generality, we may assume that $v_{0} \equiv 0$. We set $w(t):=\left(-\Delta_{x}\right)^{-\frac{1+\kappa}{2}} v(t)$. Then, this function solves the equation

$$
\partial_{t} w-\Delta_{x} w=\bar{\xi}(t):=\left(-\Delta_{x}\right)^{(1-\kappa) / 2} \xi(t)
$$

Moreover, according to the anisotropic regularity theorem for the heat equation (see, e.g., [Mie]), we have

$$
\begin{aligned}
& \int_{0}^{t} e^{-\alpha(t-s)}\left(\left\|\partial_{t} w(s)\right\|_{L^{2}}^{2 /(1-\kappa)}+\|w(s)\|_{H^{2}}^{2 /(1-\kappa)}\right) d s \leq \\
& \quad \leq C \int_{0}^{t} e^{-\alpha(t-s)}\|\bar{\xi}(s)\|_{L^{2}}^{2 /(1-\kappa)} d s \leq C_{1} \int_{0}^{t} e^{-\alpha(t-s)}\|\xi(s)\|_{H^{1-\kappa}}^{2 /(1-\kappa)} d s
\end{aligned}
$$

for all sufficiently small $\alpha \geq 0$. Using now a proper embedding theorem for anisotropic Sobolev spaces, we infer

$$
\|w(t)\|_{H^{1+\kappa}} \leq C\left(\|w\|_{L^{2 /(1-\kappa)}\left([t-1, t], H^{2}\right)}+\left\|\partial_{t} w\right\|_{L^{2 /(1-\kappa)}\left([t-1, t], L^{2}\right)}\right),
$$

where the constant $C$ is independent of $t$ (as usual, we extend the function $w(t)$ by zero for $t<0$ ). Combining (1.55) with (1.54) and using the fact that $\|v(t)\|_{L^{2}} \leq$ $C\|w(t)\|_{H^{1+\kappa}}$, we finally obtain 


$$
\begin{aligned}
\left\|e^{\alpha t} v(t)\right\|_{L^{2}}^{2 /(1-\kappa)} \leq C \| e^{\alpha t} w(t) & \|_{H^{1+\kappa}}^{2 /(1-\kappa)} \leq \\
& \leq C_{1} \int_{0}^{t} e^{\alpha^{\prime} s}\left(\left\|\partial_{t} w(s)\right\|_{L^{2}}^{2 /(1-\kappa)}+\|w(s)\|_{H^{2}}^{2 /(1-\kappa)}\right) d s,
\end{aligned}
$$

so that, for $\alpha$ small enough,

$$
\|v(t)\|_{L^{2}}^{2 /(1-\kappa)} \leq C \int_{0}^{t} e^{-\alpha^{\prime}(t-s)}\|\xi(s)\|_{H^{1-\kappa}}^{2 /(1-\kappa)} d s
$$

which finishes the proof of the lemma.

We now apply Lemma 1.8 to the first equation of (1.42) which we rewrite in the form (1.51) with $v(t):=\bar{\theta}(t)+\left[H\left(u_{1}(t)\right)-H\left(u_{2}(t)\right)\right]$ and $\xi(t):=H\left(u_{1}(t)\right)-H\left(u_{2}(t)\right)$. Indeed, since $H^{\prime}$ and $\left\|u_{i}\right\|_{L^{2}\left([0, T], H^{1}\right)}$ are uniformly bounded, we have

$$
\int_{0}^{T}\|\xi(t)\|_{H^{1}}^{2} d t \leq C T .
$$

On the other hand, according to (1.50), there holds

$$
\|\xi(t)\|_{L^{2}} \leq C e^{K t}\left(\|\bar{u}(0)\|_{L^{2}}+\|\bar{\theta}(0)\|_{L^{2}}\right) .
$$

Combining (1.57) and (1.58) and using an appropriate interpolation inequality, we infer

$$
\int_{0}^{T}\|\xi(t)\|_{H^{1-\kappa}}^{2 /(1-\kappa)} d t \leq C_{1} e^{K T}\left(\|\bar{u}(0)\|_{L^{2}}+\|\bar{\theta}(0)\|_{L^{2}}\right)
$$

and, consequently, Lemma 1.8 gives

$$
\left\|\bar{\theta}(t)+\left[H\left(u_{1}(t)\right)-H\left(u_{2}(t)\right)\right]\right\|_{L^{2}}^{2 /(1-\kappa)} \leq C_{2} e^{K t}\left(\|\bar{u}(0)\|_{L^{2}}+\|\bar{\theta}(0)\|_{L^{2}}\right) .
$$

This estimate, together with (1.50), give (1.41) and finish the proof of Theorem 1.3.

Theorem 1.1 allows to define the solving semigroup $S_{t}$ associated with problem (1.1) by the following standard expression:

$$
S_{t}\left(\theta_{0}, u_{0}\right):=(\theta(t), u(t)), \quad S_{t}: \Psi \rightarrow \Psi,
$$

where $\Psi:=\left\{(\theta, u) \in\left[H^{2}(\Omega) \cap H_{0}^{1}(\Omega)\right]^{2},\|u\|_{L^{\infty}}<1\right\}$ and $(\theta(t), u(t))$ is the unique solution of problem (1.1) with initial data $\left(\theta_{0}, u_{0}\right)$. Moreover, since the constants $C$ and $K$ in estimate (1.41) depend only on the $L^{2}$-norms of the initial data, we 
can extend in a unique way (by continuity) the semigroup (1.60) to the Hölder continuous semigroup $\hat{S}_{t}$ acting on the closure $\Phi$ of $\Psi$ in $\left[L^{2}(\Omega)\right]^{2}$, i.e.,

$$
\hat{S}_{t}: \Phi \rightarrow \Phi, \quad \Phi:=L^{2}(\Omega) \times\left\{u \in L^{\infty}(\Omega),\|u\|_{L^{\infty}} \leq 1\right\},
$$

and, for every $\left(\theta_{0}, u_{0}\right) \in \Phi$, we have

$$
\hat{S}_{t}\left(\theta_{0}, u_{0}\right):=\left[L^{2}(\Omega)\right]^{2}-\lim _{n \rightarrow \infty} S_{t}\left(\theta_{0}^{n}, u_{0}^{n}\right),
$$

where $\left(\theta_{0}^{n}, u_{0}^{n}\right)$ is an arbitrary sequence belonging to $\Psi$ and converging to $\left(\theta_{0}, u_{0}\right)$ in the topology of $\left[L^{2}(\Omega)\right]^{2}$. Furthermore, thanks to Theorem 1.2, we have the smoothing property

$$
\hat{S}_{t}: \Phi \rightarrow \Psi, \quad \forall t>0,
$$

and it is not difficult to verify, using the definition of the semigroup $\hat{S}_{t}$ and the smoothing property $(1.63)$, that every trajectory $(\hat{\theta}(t), \hat{u}(t))$ of this semigroup belongs to the space $C\left([0, T],\left[L^{2}(\Omega)\right]^{2}\right)$ and satisfies system (1.1) in the sense of distributions. Thus, we have proven the following result.

Corollary 1.1. Let the assumptions of Theorem 1.1 hold. Then, for every initial data $\left(\theta_{0}, u_{0}\right) \in \Phi$, equation (1.1) possesses a unique solution $(\hat{\theta}(t), \hat{u}(t))$ which belongs to $C\left([0, T],\left[L^{2}(\Omega)\right]^{2}\right)$ and belongs to $\Psi$ for every $t>0$. Moreover, this solution satisfies $(\hat{\theta}(t), \hat{u}(t))=\hat{S}_{t}\left(\theta_{0}, u_{0}\right)$.

In order to simplify the notation, we write below $S_{t}$ instead of $\hat{S}_{t}$ when it does not lead to confusion.

We conclude this section with several additional regularity estimates for the solutions of (1.1) which are necessary to study the boundary layer term at $t=0$ in system (0.1) with small positive $\varepsilon$ (see Section 3).

Corollary 1.2. Let the assumptions of Theorem 1.1 hold and let, in addition, $g$ belong to $H^{1}(\Omega)$. Then, any solution of problem (1.1) satisfies the following smoothing property

$$
\begin{aligned}
\mathbb{D}[u(t)]+\|u(t)\|_{H^{3}}+\|\theta(t)\|_{H^{3}}+\left\|\partial_{t} u(t)\right\|_{H^{1}}+\left\|\partial_{t} \theta(t)\right\|_{H^{1}} \leq \\
\leq Q\left(t^{-1}+\|\theta(0)\|_{L^{2}}\right) e^{-\alpha t}+Q\left(\|g\|_{L^{\infty} \cap H^{1}}\right), t>0,
\end{aligned}
$$

where the positive constant $\alpha$ and the monotonic function $Q$ are independent of $t>0$ and of the solution $(\theta, u)$. Moreover, if the initial data $\left(\theta_{0}, u_{0}\right)$ satisfies

$$
\mathbb{D}[u(0)]+\left\|u_{0}\right\|_{H^{3}}+\left\|\theta_{0}\right\|_{H^{3}}<\infty,\left.\quad \Delta_{x} \theta_{0}\right|_{\partial \Omega}=0, \quad-\left.\Delta_{x} u_{0}\right|_{\partial \Omega}=\left.g\right|_{\partial \Omega},
$$

then, the following estimate holds

$$
\begin{aligned}
\mathbb{D}[u(t)]+ & \|u(t)\|_{H^{3}}+\|\theta(t)\|_{H^{3}}+\left\|\partial_{t} u(t)\right\|_{H^{1}}+\left\|\partial_{t} \theta(t)\right\|_{H^{1}} \leq \\
& \leq Q\left(\mathbb{D}[u(0)]+\|\theta(0)\|_{H^{3}}+\left\|u_{0}\right\|_{H^{3}}\right) e^{-\alpha t}+Q\left(\|g\|_{L^{\infty} \cap H^{1}}\right), \quad t \geq 0 .
\end{aligned}
$$

Indeed, since, due to Theorems 1.1 and 1.2, the solution $(\theta(t), u(t))$ is strictly separated from the singular points $r= \pm 1$ of the nonlinearity $f$, then estimates (1.64) and (1.66) are immediate consequences of standard parabolic regularity estimates (see, e.g., [LSU]). 
Corollary 1.3. Let the assumptions of Corollary 1.2 hold. Then, for every initial data $\left(\theta_{0}, u_{0}\right)$ satisfying (1.65), the following estimate is valid

$$
\begin{aligned}
&\left\|\partial_{t}^{2} u(t)\right\|_{H^{-1}}^{2}+\int_{t}^{t+1}\left(\left\|\partial_{t}^{2} u(s)\right\|_{L^{2}}^{2}+\left\|\left(-\Delta_{x}\right)^{-1} \partial_{t}^{3} u(s)\right\|_{L^{2}}^{2}\right) d s \leq \\
& \leq Q\left(\mathbb{D}[u(0)]+\left\|u_{0}\right\|_{H^{3}}+\left\|\theta_{0}\right\|_{H^{3}}\right) e^{-\alpha t}+Q\left(\|g\|_{L^{\infty} \cap H^{1}}\right),
\end{aligned}
$$

where the constant $\alpha>0$ and the monotonic function $Q$ are independent of the solution $(\theta, u)$.

Estimate (1.67) is also a standard corollary of parabolic regularity estimates. We thus leave its rigorous proof to the reader.

\section{$\S 2$ THE CASE $\varepsilon>0$ : UNIFORM A PRIORI \\ ESTIMATES AND EXISTENCE OF SOLUTIONS}

In this section, we study equation $(0.1)$ for positive $\varepsilon$. In that case, the second equation of (0.1) is hyperbolic and we have neither the monotonicity property nor the maximum principle for this equation. Nevertheless, we will show below, using some kind of a perturbation technique, that this equation possesses a unique strong solution $(\theta(t), u(t))$ if $\varepsilon \ll 1$ is small enough and the $\mathcal{E}^{1}$-energy of the initial data is not too large and obtain some uniform (with respect to $\varepsilon \rightarrow 0$ ) estimates on this solution. The main result of this section is the following theorem.

Theorem 2.1. Let the nonlinearities $H$ and $f$ satisfy assumptions (0.2) and $g$ belong to $L^{\infty}(\Omega)$. Then, there exist a positive constant $\varepsilon_{0}$ and a monotonic decreasing function $R:\left(0, \varepsilon_{0}\right] \rightarrow \mathbb{R}_{+}$such that

$$
\lim _{\varepsilon \rightarrow 0} R(\varepsilon)=+\infty
$$

and, for every $\varepsilon \leq \varepsilon_{0}$ and every initial data $\left(\theta(0), \xi_{u}(0)\right)$ satisfying

$$
\mathbb{D}[u(0)]+\|\theta(0)\|_{H^{2}}+\left\|\xi_{u}(0)\right\|_{\mathcal{E}^{1}(\varepsilon)} \leq R(\varepsilon),
$$

there exists a unique global solution $\left(\theta(t), \xi_{u}(t)\right)$ of problem $(0.1)$ which satisfies the following estimate

$$
\begin{aligned}
\mathbb{D}[u(t)]+\|\theta(t)\|_{H^{2}}^{2}+ & \left\|\partial_{t} \theta(t)\right\|_{L^{2}}^{2}+\left\|\xi_{u}(t)\right\|_{\mathcal{E}^{1}(\varepsilon)}^{2}+ \\
& +\int_{0}^{t} e^{-\alpha(t-s)}\left\|\partial_{t} u(s)\right\|_{H^{1}}^{2} d s \leq \\
\leq & Q\left(\mathbb{D}[u(0)]+\|\theta(0)\|_{H^{2}}+\left\|\xi_{u}(0)\right\|_{\mathcal{E}^{1}(\varepsilon)}\right) e^{-\alpha t}+Q\left(\|g\|_{L^{\infty}}\right),
\end{aligned}
$$

where the positive constant $\alpha$ and the monotonic function $Q$ are independent of $\varepsilon$.

Proof. We first derive the a priori estimate $(2.3)$, assuming that $\left(\theta(t), \xi_{u}(t)\right)$ is a sufficiently regular solution of $(0.1)$ which is separated from the singular points of $f$, i.e.,

$$
\|u\|_{L^{\infty}\left(\mathbb{R}_{+} \times \Omega\right)}<1 .
$$

We start with a classical $\mathcal{E}$-energy estimate. 
Lemma 2.1. Let the above assumptions hold. Then, the solution $\left(\theta(t), \xi_{u}(t)\right)$ satisfies the following estimate

$$
\begin{aligned}
& \varepsilon\left\|\partial_{t} u(t)\right\|_{L^{2}}^{2}+\|u(t)\|_{H^{1}}^{2}+\|\theta(t)\|_{L^{2}}^{2}+\int_{0}^{t} e^{-\alpha(t-s)}\left\|\partial_{t} u(s)\right\|_{L^{2}}^{2} d s \leq \\
& \leq Q\left(\mathbb{D}[u(0)]+\varepsilon\left\|\partial_{t} u(0)\right\|_{L^{2}}^{2}+\|u(0)\|_{H^{1}}^{2}+\|\theta(0)\|_{L^{2}}^{2}\right) e^{-\alpha t}+Q\left(\|g\|_{L^{2}}\right),
\end{aligned}
$$

where the positive constant $\alpha$ and the monotonic function $Q$ are independent of $\varepsilon$.

Proof. Multiplying the first and the second equations of $(0.1)$ by $\theta(t)$ and $\partial_{t} u(t)+$ $\beta u(t)$ respectively (where $\beta$ is a small positive parameter which will be fixed below), summing the equations that we obtain and integrating over $\Omega$, we have

$$
\begin{gathered}
\frac{1}{2} \frac{d}{d t}\left[\varepsilon\left\|\partial_{t} u(t)\right\|_{L^{2}}^{2}+\left\|\nabla_{x} u(t)\right\|_{L^{2}}^{2}+2 \beta \varepsilon\left(u(t), \partial_{t} u(t)\right)_{L^{2}}+\beta\|u(t)\|_{L^{2}}^{2}+\right. \\
\left.+\|\theta(t)\|_{L^{2}}^{2}+2(F(u(t)), 1)_{L^{2}}\right]+ \\
+(1-\varepsilon \beta)\left\|\partial_{t} u(t)\right\|_{L^{2}}^{2}+\beta\left\|\nabla_{x} u(t)\right\|_{L^{2}}^{2}+\left\|\nabla_{x} \theta(t)\right\|_{L^{2}}^{2}+ \\
+\beta(f(u(t)), u(t))_{L^{2}}=\left(g, \partial_{t} u(t)+\beta u(t)\right)_{L^{2}}+\beta\left(H^{\prime}(u(t)) \theta(t), u(t)\right)_{L^{2}}
\end{gathered}
$$

Using now estimate (1.7), fixing $\beta>0$ small enough, applying Gronwall's inequality to (2.6) and arguing as in the proof of Lemma 1.1, we deduce estimate (2.5) and finish the proof of Lemma 2.1 (see also [GrP1] and [GrP2]). We mention that, in contrast to [GrP1] and [GrP2], we now have, a priori, $\|u(t)\|_{L^{\infty}} \leq 1$ and, consequently, no growth restrictions on $H$ are required in order to handle the term $\left(H^{\prime}(u) \theta, u\right)_{L^{2}}$.

In a next step, we obtain an $H^{1}$-estimate for the $\theta$-component of the solution of (0.1).

Lemma 2.2. Let the above assumptions hold. Then, the following estimate is valid

$$
\begin{aligned}
\|\theta(t)\|_{H^{1}}^{2}+\int_{0}^{t} e^{-\alpha(t-s)}\left(\left\|\partial_{t} \theta(s)\right\|_{L^{2}}^{2}+\|\theta(s)\|_{H^{2}}^{2}\right) d s \leq \\
\quad \leq Q\left(\varepsilon\left\|\partial_{t} u(0)\right\|_{L^{2}}^{2}+\|u(0)\|_{H^{1}}^{2}+\|\theta(0)\|_{H^{1}}^{2}\right) e^{-\alpha t}+Q\left(\|g\|_{L^{2}}\right),
\end{aligned}
$$

where the positive constant $\alpha$ and the monotonic function $Q$ are independent of $\varepsilon$.

Indeed, since $\|u(t)\|_{L^{\infty}} \leq 1$ and the function $H$ is regular (we recall that, in contrast to the nonlinearity $f$, the function $H$ does not have any singularity at $r= \pm 1$ ), then

$$
\left\|\partial_{t} H(u(t))\right\|_{L^{2}} \leq C\left\|\partial_{t} u(t)\right\|_{L^{2}}
$$

Estimate (2.7) is now an immediate consequence of (2.5), (2.8) and the classical $L^{2}$-regularity estimate $\left(1.9^{\prime}\right)$ for the heat equation applied to the first component of system (0.1). 
In order to obtain a priori estimates for the $\mathcal{E}^{1}$-norm of the solutions of problem (0.1), we will compare them with the solutions of the limit parabolic system (which corresponds to $\varepsilon=0$ in (0.1)), taking $\varepsilon$ small. To this end, it is more convenient, following $[\mathrm{Z}]$, to modify slightly the second equation of (0.1) with $\varepsilon=0$ and to consider the following auxiliary system:

$$
\left\{\begin{array}{l}
\partial_{t}\left(\theta_{0}+H\left(u_{0}\right)\right)-\Delta_{x} \theta_{0}=0,\left.\quad \theta\right|_{\partial \Omega}=0,\left.\quad \theta_{0}\right|_{t=0}=\theta(0),\left.u_{0}\right|_{t=0}=u(0), \\
\partial_{t} u_{0}-\Delta_{x} u_{0}+f\left(u_{0}\right)-H^{\prime}\left(u_{0}\right) \theta_{0}+L u_{0}=h_{u}(t),\left.\quad u_{0}\right|_{\partial \Omega}=0
\end{array}\right.
$$

where $L$ is a sufficiently large positive parameter which will be specified below and $h_{u}(t):=g+L u(t)$. The following Lemma is the analogue of estimate (1.3) for the auxiliary problem (2.9).

Lemma 2.3. Let the nonlinearities $H$ and $f$ satisfy assumptions (0.2), $g$ belong to $L^{\infty}(\Omega)$ and the function $u(t)$ satisfy $\|u(t)\|_{L^{\infty}} \leq 1$. Then, equation (2.9) possesses a unique solution $\left(\theta_{0}(t), u_{0}(t)\right)$ which satisfies the estimate

$$
\begin{aligned}
\mathbb{D}\left[u_{0}(t)\right]+\| \theta_{0}(t) & \left\|_{H^{2}}^{2}+\right\| u_{0}(t) \|_{H^{2}}^{2} \leq \\
& \leq Q\left(\mathbb{D}[u(0)]+\|\theta(0)\|_{H^{2}}^{2}+\|u(0)\|_{H^{2}}^{2}\right) e^{-\alpha t}+Q\left(\|g\|_{L^{\infty}}\right),
\end{aligned}
$$

where the positive constant $\alpha$ and the monotonic function $Q$ depend on $L$.

The proof of this Lemma is completely analogous to that of Theorem 1.1 and is left to the reader.

The next lemma shows that the solutions $\left(\theta(t), \xi_{u}(t)\right)$ of $(0.1)$ are, in some sense, close to the solutions $\left(\theta_{0}(t), u_{0}(t)\right)$ of the auxiliary problem $(2.9)$ if $\varepsilon>0$ is small enough and $L$ is large enough.

Lemma 2.4. Let the above assumptions hold and let $\left(\theta(t), \xi_{u}(t)\right)$ be a solution of problem (0.1). Then, there exists a sufficiently large, but independent of $\varepsilon$, constant $L$ such that

$$
\begin{aligned}
& \left\|u(t)-u_{0}(t)\right\|_{L^{2}}^{2}+\int_{0}^{t} e^{-\alpha(t-s)}\left\|\theta(s)-\theta_{0}(s)\right\|_{L^{2}}^{2} d s \leq \\
\leq & \varepsilon\left(Q\left(\mathbb{D}[u(0)]+\|\theta(0)\|_{H^{1}}^{2}+\varepsilon\left\|\partial_{t} u(0)\right\|_{L^{2}}^{2}+\|u(0)\|_{H^{1}}^{2}\right) e^{-\alpha t}+Q\left(\|g\|_{L^{\infty}}\right)\right),
\end{aligned}
$$

where $\left(\theta_{0}(t), u_{0}(t)\right)$ is a solution of problem (2.9) and the positive constant $\alpha$ and the monotonic function $Q$ are independent of $\varepsilon$.

Proof of Lemma 2.4. We set $\bar{\theta}(t):=\theta(t)-\theta_{0}(t)$ and $\bar{u}(t):=u(t)-u_{0}(t)$. These functions satisfy the following equations:

$$
\left\{\begin{aligned}
\partial_{t} \bar{\theta}-\Delta_{x} \bar{\theta}=-\partial_{t}\left(H(u(t))-H\left(u_{0}(t)\right)\right),\left.\quad \bar{\theta}\right|_{t=0}=0,\left.\quad \bar{u}\right|_{t=0}=0, \\
\partial_{t} \bar{u}-\Delta_{x} \bar{u}+\left[f(u)-f\left(u_{0}\right)\right]+L \bar{u}= \\
=-\varepsilon \partial_{t}^{2} u(t)+\left[H^{\prime}(u(t)) \theta(t)-H^{\prime}\left(u_{0}(t)\right) \theta_{0}(t)\right] .
\end{aligned}\right.
$$


Applying Lemma 1.7 to the first equation of (2.12), we have

$$
\int_{0}^{t} e^{-\alpha(t-s)}\|\bar{\theta}(s)\|_{L^{2}}^{2} d s \leq C_{1} \int_{0}^{t} e^{-\alpha(t-s)}\|\bar{u}(s)\|_{L^{2}}^{2} d s
$$

where the constant $C_{1}$ is independent of $t$.

We then study the second equation of (2.12). To this end, we multiply this equation by $\bar{u}(t)$ and integrate over $\Omega$. Then, noting that $f^{\prime}(r) \geq-K$ and using estimate (1.47) (in which we replace $\theta_{1}$ and $\theta_{2}$ by $\theta$ and $\theta_{0}$ respectively), we have

$$
\begin{aligned}
& \frac{1}{2} \partial_{t}\|\bar{u}(t)\|_{L^{2}}^{2}+\frac{1}{2}\left\|\nabla_{x} \bar{u}(t)\right\|_{L^{2}}^{2}+ \\
& +\left(L-K-C_{\mu}\left(1+\|\theta(t)\|_{L^{2}}^{4}\right)\right)\|\bar{u}(t)\|_{L^{2}}^{2} \leq-\varepsilon\left(\partial_{t}^{2} u(t), \bar{u}(t)\right)_{L^{2}}+\mu\|\bar{\theta}(t)\|_{L^{2}}^{2},
\end{aligned}
$$

where $\mu>0$ is arbitrary and the constant $C_{\mu}$ is independent of $(\theta, u)$ and $\left(\theta_{0}, u_{0}\right)$. We estimate the first term in the right-hand side of (2.14) as follows:

$$
-\varepsilon\left(\partial_{t}^{2} u(t), \bar{u}(t)\right)_{L^{2}} \leq-\varepsilon \partial_{t}\left(\partial_{t} u(t), \bar{u}(t)\right)+C \varepsilon\left(\left\|\partial_{t} u(t)\right\|_{L^{2}}^{2}+\left\|\partial_{t} u_{0}(t)\right\|_{L^{2}}^{2}\right)
$$

Inserting this estimate into (2.14), we have

$$
\begin{aligned}
\frac{1}{2} \partial_{t}\left[\|\bar{u}(t)\|_{L^{2}}^{2}\right. & \left.+2 \varepsilon\left(\partial_{t} u(t), \bar{u}(t)\right)_{L^{2}}\right]+C\left[\|\bar{u}(t)\|_{L^{2}}^{2}+2 \varepsilon\left(\partial_{t} u(t), \bar{u}(t)\right)_{L^{2}}\right]+ \\
& +\left(L-K-C_{\mu}-C^{\prime}\|\theta(t)\|_{L^{2}}^{4}\right)\|\bar{u}(t)\|_{L^{2}}^{2} \leq \\
& \leq \mu\|\bar{\theta}(t)\|_{L^{2}}^{2}+C^{\prime \prime} \varepsilon\left(\left\|\partial_{t} u(t)\right\|_{L^{2}}^{2}+\left\|\partial_{t} u_{0}(t)\right\|_{L^{2}}^{2}\right)
\end{aligned}
$$

where all the constants are independent of $L$ and $\varepsilon$. We recall that, due to Lemma 2.2 ,

$$
\|\theta(t)\|_{L^{2}}^{2} \leq Q_{1} e^{-\alpha t}+Q_{0}
$$

where $Q_{1}=Q_{1}\left(\varepsilon\left\|\partial_{t} u(0)\right\|_{L^{2}}^{2}+\|u(0)\|_{H^{1}}^{2}+\|\theta(0)\|_{H^{1}}^{2}\right)$ and $Q_{0}=Q_{0}\left(\|g\|_{L^{2}}\right)$. Thus, fixing $L=L_{\mu}$ so that $L-K-C_{\mu}-C^{\prime} Q_{0}^{2} \geq 0$, applying Gronwall's inequality to (2.16) and using estimate (2.13) (in order to estimate the term containing $\bar{\theta}(t)$ ) and estimates (2.5) and (2.10) (in order to estimate the integrals of the $L^{2}$-norms of $\partial_{t} u$ and $\left.\partial_{t} u_{0}\right)$, we have

$$
\begin{aligned}
& \|\bar{u}(t)\|_{L^{2}}^{2} \leq \\
& \leq \varepsilon\left(Q_{\mu}\left(\varepsilon\left\|\partial_{t} u(0)\right\|_{L^{2}}^{2}+\|u(0)\|_{H^{1}}^{2}+\|\theta(0)\|_{L^{2}}^{2}\right) e^{-\alpha t}+Q_{\mu}\left(\|g\|_{L^{2}}\right)\right)+ \\
& \quad+C \mu \int_{0}^{t} e^{-\alpha(t-s)}\|\bar{u}(s)\|_{L^{2}}^{2} d s+C Q_{1}^{2} e^{-\alpha t} \int_{0}^{t} e^{-\alpha(t-s)}\|\bar{u}(s)\|_{L^{2}}^{2} d s
\end{aligned}
$$

where the positive constants $C$ and $\alpha$ and the monotonic function $Q_{\mu}$ are independent of $\varepsilon$ and $\left(\theta(0), \xi_{u}(0)\right)$ and where the constants $C$ and $\alpha$ are also independent 
of $\mu$. Fixing finally the parameter $\mu>0$ such that $C \mu<\alpha$ and applying once more Gronwall's inequality to relation (2.18), we finally find

$$
\|\bar{u}(t)\|_{L^{2}}^{2} \leq \varepsilon\left(Q\left(\varepsilon\left\|\partial_{t} u(0)\right\|_{L^{2}}^{2}+\|u(0)\|_{H^{1}}^{2}+\|\theta(0)\|_{L^{2}}^{2}\right) e^{-\alpha t}+Q\left(\|g\|_{L^{2}}\right)\right),
$$

for some (new) positive constant $\alpha$ and monotonic function $Q$ which are independent of $\varepsilon$. Estimate (2.11) is now an immediate consequence of (2.19) and (2.13) and Lemma 2.4 is proven.

We are now ready to obtain $\mathcal{E}^{1}$-estimates on the solutions of $(0.1)$. To this end, we multiply the second equation of $(0.1)$ by $-\Delta_{x}\left(\partial_{t} u(t)+\beta u(t)\right)$, where $\beta>0$ is a sufficiently small (but independent of $\varepsilon$ ) constant, and integrate over $\Omega$. Then, after standard transformations, we have

$$
\begin{aligned}
\frac{d}{d t} E_{u}(t)+\beta E_{u}(t)+\beta / 2\left(\| \Delta_{x} u(t)\right. & \left.\left\|_{L^{2}}^{2}+\right\| \nabla_{x} \partial_{t} u(t) \|_{L^{2}}^{2}\right) \leq \\
& \leq C\left(\|f(u(t))\|_{H^{1}}^{2}+\left\|H^{\prime}(u(t)) \theta(t)\right\|_{H^{1}}^{2}\right),
\end{aligned}
$$

where

$$
\begin{aligned}
E_{u}(t):=\varepsilon\left\|\nabla_{x} \partial_{t} u(t)\right\|_{L^{2}}^{2}+\beta\left\|\nabla_{x} u(t)\right\|_{L^{2}}^{2}+\left\|\Delta_{x} u(t)\right\|_{L^{2}}^{2}- & \\
& -2\left(g, \Delta_{x} u(t)\right)_{L^{2}}+\beta \varepsilon\left(\nabla_{x} u(t), \nabla_{x} \partial_{t} u(t)\right)_{L^{2}} .
\end{aligned}
$$

We now estimate the right-hand side of (2.20). We first note that the second term in the right-hand side of (2.20) is easy to estimate, due to estimates (2.5) and (2.7) and to the interpolation inequality $\left\|\nabla_{x} u\right\|_{L^{3}}^{2} \leq C\|u\|_{H^{1}}\|u\|_{H^{2}}$. Indeed,

$$
\begin{aligned}
& \left\|H^{\prime}(u(t)) \theta(t)\right\|_{H^{1}}^{2} \leq \\
& \leq C\|\theta(t)\|_{H^{1}}^{2}+C\left\|\theta(t) \cdot \nabla_{x} u(t)\right\|_{L^{2}}^{2} \leq C\|\theta(t)\|_{H^{1}}^{2}\left(1+\left\|\nabla_{x} u(t)\right\|_{L^{3}}^{2}\right) \leq \\
& \leq \frac{\beta}{2}\left\|\Delta_{x} u(t)\right\|_{L^{2}}^{2}+C_{1}\left(1+\|\theta(t)\|_{H^{1}}^{4}\right)\left(1+\|u(t)\|_{H^{1}}^{2}\right) \leq \frac{\beta}{2}\left\|\Delta_{x} u(t)\right\|_{L^{2}}^{2}+ \\
& \quad+Q\left(\varepsilon\left\|\partial_{t} u(0)\right\|_{L^{2}}^{2}+\|u(0)\|_{H^{1}}^{2}+\|\theta(0)\|_{H^{1}}^{2}\right) e^{-\alpha t}+Q\left(\|g\|_{L^{2}}\right),
\end{aligned}
$$

where $\beta$ is the same as in (2.20) and the positive constant $\alpha$ and the monotonic function $Q$ are independent of $\varepsilon$. So, it only remains to estimate the first term in the right-hand side of (2.20). To this end, we will essentially use the fact that, due to Lemma 2.4, the function $u(t)$ is close to the regular function $u_{0}(t)$ if $\varepsilon>0$ is small enough. Moreover, noting that the function $f$ is regular inside the interval $(-1,1)$, 
we have

$$
\begin{aligned}
& \left\|f\left(u_{0}(t)\right)-f(u(t))\right\|_{H^{1}(\Omega)}^{2}= \\
& =\left\|\nabla_{x}\left(\int_{0}^{1} f^{\prime}\left(s u_{0}(t)+(1-s) u(t)\right) d s \cdot\left(u_{0}(t)-u(t)\right)\right)\right\|_{L^{2}}^{2} \leq \\
& \leq \sup _{s \in[0,1]}\left[\left\|f^{\prime}\left(s u_{0}+(1-s) u\right)\right\|_{L^{\infty}}^{2}+\right. \\
& \left.+\left\|f^{\prime \prime}\left(s u_{0}+(1-s) u\right)\right\|_{L^{\infty}}^{2}\left(1+\left\|u_{0}(t)\right\|_{H^{2}}^{2}+\|u(t)\|_{H^{2}}^{2}\right)\right] \cdot\left\|u(t)-u_{0}(t)\right\|_{H^{1}}^{2} \leq \\
& \leq \sup _{s \in[0,1]} \mathbb{M}_{f}\left(\frac{1}{1-\left\|s u_{0}+(1-s) u\right\|_{L^{\infty}}}\right) \times \\
& \times\left(1+\left\|u_{0}(t)\right\|_{H^{2}}^{2}+\|u(t)\|_{H^{2}}^{2}\right)\left\|u(t)-u_{0}(t)\right\|_{H^{1}}^{2} \leq \\
& \mathbb{M}_{f}\left(\frac{1}{1-\left\|u_{0}(t)\right\|_{L^{\infty}}-\left\|u(t)-u_{0}(t)\right\|_{L^{\infty}}}\right)\left(1+\left\|u_{0}(t)\right\|_{H^{2}}^{2}+\|u(t)\|_{H^{2}}^{2}\right) \times \\
& \times\left\|u(t)-u_{0}(t)\right\|_{H^{1}}^{2},
\end{aligned}
$$

for some smooth monotonic function $\mathbb{M}_{f}(z)$ depending only on $f$ (due to condition $\left.(0.2)_{3}, \lim _{z \rightarrow \infty} \mathbb{M}_{f}(z)=\infty\right)$. Using now the interpolation inequalities

$\left\|u-u_{0}\right\|_{H^{1}} \leq C\left\|u-u_{0}\right\|_{L^{2}}^{1 / 2}\left\|u-u_{0}\right\|_{H^{2}}^{1 / 2}, \quad\left\|u-u_{0}\right\|_{L^{\infty}} \leq C\left\|u-u_{0}\right\|_{L^{2}}^{1 / 4}\left\|u-u_{0}\right\|_{H^{2}}^{3 / 4}$,

estimate (2.11) for the $L^{2}$-norm of $u-u_{0}$, the obvious estimate

$$
\left\|u(t)-u_{0}(t)\right\|_{H^{2}}^{2} \leq C\left(E_{u}(t)+\left\|u_{0}(t)\right\|_{H^{2}}^{2}+\|g\|_{L^{2}}^{2}\right)
$$

and estimate (2.10) in order to estimate the norms of $u_{0}(t)$, we have

$$
\begin{aligned}
1-\|u(t)\|_{L^{\infty}} \geq 1-\left\|u_{0}(t)\right\|_{L^{\infty}}-\left\|u(t)-u_{0}(t)\right\|_{L^{\infty}} \geq \\
\geq\left(\bar{Q}+Q_{0} e^{-\alpha t}\right)^{-1}-\varepsilon^{1 / 8}\left(\bar{Q}+Q_{0} e^{-\alpha t}\right)\left(1+E_{u}(t)\right)
\end{aligned}
$$

and, consequently,

$$
\begin{aligned}
& \|f(u(t))\|_{H^{1}}^{2} \leq \\
& \leq Q_{0} \varepsilon^{1 / 2}\left(1+E_{u}(t)\right)^{2} \mathbb{M}_{f}\left(\frac{1}{\left(\bar{Q}+Q_{0}\right)^{-1}-\varepsilon^{1 / 8}\left(\bar{Q}+Q_{0}\right)\left(1+E_{u}(t)\right)}\right)+ \\
& +Q_{0} e^{-\alpha t}+\bar{Q},
\end{aligned}
$$

where the monotonic increasing functions

$$
Q_{0}=Q_{0}\left(\mathbb{D}[u(0)]+\|\theta(0)\|_{H^{2}}+\left\|\xi_{u}(0)\right\|_{\mathcal{E}^{1}(\varepsilon)}+\|g\|_{L^{\infty}}\right)
$$


and $\bar{Q}=\bar{Q}\left(\|g\|_{L^{\infty}}\right)$ and the positive constant $\alpha$ are independent of $\varepsilon$ (obviously, estimate $(2.24)$ has a sense only if $\left.\left(\bar{Q}+Q_{0}\right)^{-1}-\varepsilon^{1 / 8}\left(\bar{Q}+Q_{0}\right)\left(1+E_{u}(t)\right)>0\right)$.

Inserting estimates (2.21) and (2.24) into the right-hand side of (2.20), we finally deduce that the function $E_{u}(t)$ satisfies the following differential inequality:

$$
\begin{aligned}
& \frac{d}{d t} E_{u}(t)+\beta E_{u}(t) \leq \\
\leq & Q_{0} \varepsilon^{1 / 2}\left(1+E_{u}(t)\right)^{2} \mathbb{M}_{f}\left(\frac{1}{\left(\bar{Q}+Q_{0}\right)^{-1}-\varepsilon^{1 / 8}\left(\bar{Q}+Q_{0}\right)\left(1+E_{u}(t)\right)}\right)+ \\
& +2 Q_{0} e^{-\alpha t}+2 \bar{Q},
\end{aligned}
$$

where we can assume, without loss of generality, that $\alpha<\beta$. The following Lemma shows that inequality (2.25) allows to obtain a dissipative estimate for $E_{u}(t)$ if the initial values $\theta(0)$ and $\xi_{u}(0)$ are not too large.

Lemma 2.5. Let the initial data $\left(\theta(0), \xi_{u}(0)\right)$ satisfy the inequality

$$
\mathbb{D}[u(0)]+\|\theta(0)\|_{H^{2}}+\left\|\xi_{u}(0)\right\|_{\mathcal{E}^{1}(\varepsilon)}+\|g\|_{L^{2}} \leq R(\varepsilon),
$$

where $R=R(\varepsilon)$ solves the equation

$$
\begin{gathered}
\bar{Q}\left(\|g\|_{L^{\infty}}\right)= \\
=Q_{0}(R) \varepsilon^{1 / 2}\left(1+2(\beta-\alpha)^{-1} Q_{0}(R)+3 \beta^{-1} \bar{Q}\right)^{2} \times \\
\times \mathbb{M}_{f}\left(\frac{1}{\left(\bar{Q}+Q_{0}(R)\right)^{-1}-\varepsilon^{1 / 8}\left(\bar{Q}+Q_{0}(R)\right)\left(1+2(\beta-\alpha)^{-1} Q_{0}(R)+3 \beta^{-1} \bar{Q}\right)}\right) .
\end{gathered}
$$

Then, the following estimate is valid

$$
\begin{aligned}
& E_{u}(t) \leq E_{0}(t):= \\
& \begin{aligned}
=2(\beta-\alpha)^{-1} Q_{0}\left(\mathbb{D}[u(0)]+\|\theta(0)\|_{H^{2}}+\left\|\xi_{u}(0)\right\|_{\mathcal{E}^{1}(\varepsilon)}\right. & \left.+\|g\|_{L^{2}}\right) e^{-\alpha t}+ \\
& +3 \beta^{-1} \bar{Q}\left(\|g\|_{L^{\infty}}\right) .
\end{aligned}
\end{aligned}
$$

Proof of Lemma 2.5. Since the right-hand side of (2.27) is monotonic with respect to $R$, then the function $E_{0}(t)$ satisfies the inequality

$$
\begin{aligned}
& \frac{d}{d t} E_{0}(t)+\beta E_{0}(t) \geq Q_{0} \varepsilon^{1 / 2}\left(1+E_{0}(t)\right)^{2} \times \\
& \quad \times \mathbb{M}_{f}\left(\frac{1}{\left(\bar{Q}+Q_{0}\right)^{-1}-\varepsilon^{1 / 8}\left(\bar{Q}+Q_{0}\right)\left(1+E_{0}(t)\right)}\right)+2 Q_{0} e^{-\alpha t}+2 \bar{Q}
\end{aligned}
$$

if (2.26) holds. Moreover, increasing the function $Q_{0}$ if necessary, we can assume without loss of generality that

$$
E_{u}(0) \leq E_{0}(0)
$$


Consequently, due to the comparison principle for first order differential inequalities, we have estimate (2.28) and Lemma 2.5 is proven.

Thus, due to (2.28), we have proven that, for initial data satisfying (2.26),

$$
\begin{aligned}
\varepsilon\left\|\partial_{t} u(t)\right\|_{H^{1}}^{2}+ & \|u(t)\|_{H^{2}}^{2} \leq \\
& \leq Q\left(\mathbb{D}[u(0)]+\|\theta(0)\|_{H^{2}}+\left\|\xi_{u}(0)\right\|_{\mathcal{E}^{1}(\varepsilon)}\right) e^{-\alpha t}+Q\left(\|g\|_{L^{\infty}}\right)
\end{aligned}
$$

where the positive constant $\alpha$ and the monotonic function $Q$ are independent of $\varepsilon$. It is also worth noticing that, due to equation (2.27), we have

$$
\lim _{\varepsilon \rightarrow 0} R(\varepsilon)=\infty
$$

Moreover, it follows from $(2.26)$ and $(2.27)$ that $\left(\bar{Q}+Q_{0}\right)^{-1}-\varepsilon^{1 / 8}\left(\bar{Q}+Q_{0}\right)\left(1+E_{0}(0)\right)$ is strictly positive for all initial data satisfying (2.2). Then, inserting (2.28) into the right-hand side of $(2.23)$, we verify that the solution $u(t)$ is indeed separated from the singular points $r= \pm 1$, if $\varepsilon>0$ is small enough, and the following estimate is valid:

$$
\mathbb{D}[u(t)] \leq Q\left(\mathbb{D}[u(0)]+\|\theta(0)\|_{H^{2}}+\left\|\xi_{u}(0)\right\|_{\mathcal{E}^{1}(\varepsilon)}\right) e^{-\alpha t}+Q\left(\|g\|_{L^{\infty}}\right)
$$

for some (new) positive constant $\alpha$ and monotonic function $Q$.

Thus, the most difficult part of estimate (2.3) is proven. The estimates for the other terms can be obtained in a standard way by using estimates (2.30) and (2.32). Indeed, multiplying estimate $(2.20)$ by $e^{-\beta(T-t)}$, integrating over $[0, T]$ and using (2.30) and (2.32), we have the estimate for the term $\int_{0}^{T} e^{-\beta(T-t)}\left\|\partial_{t} u(t)\right\|_{H^{1}}^{2} d t$. Having this estimate, we can interpret the term $\partial_{t} H(u)$ in the first equation of (0.1) as external forces and apply an appropriate regularity theorem for the heat equation that we obtain. Then, using also (2.30), we find the required estimate for the terms $\|\theta(t)\|_{H^{2}}^{2}$ and $\left\|\partial_{t} \theta(t)\right\|_{L^{2}}^{2}$ (see (1.17) and (1.18)). Finally, rewriting the second equation of (0.1) in the following form:

$$
\varepsilon \partial_{t}^{2} u+\partial_{t} u-\Delta_{x} u=\tilde{h}_{\theta, u}(t):=g+H^{\prime}(u(t)) \theta(t)-f(u(t))
$$

applying estimate (A.7) with $\kappa=1$ and using the estimates obtained above in order to estimate the required norms of $\tilde{h}_{\theta, u}(t)$, we can easily obtain the desired estimate for $\left\|\xi_{u}(t)\right\|_{\mathcal{E}^{1}(\varepsilon)}$ and, thus, finish the proof of the a priori estimate (2.3).

We now recall that estimate (2.3) implies, in particular, that the solution $u(t)$ is a priori strictly separated from the singular points $r= \pm 1$ of the nonlinearity $f$ and, consequently, the existence and uniqueness of this solution can be obtained exactly as in the case of regular nonlinearities, see, e.g., [GrP1] and [GrP2], and Theorem 2.1 is proven. 
Thanks to Theorem 2.1, we can define the solving semigroup $S_{t}(\varepsilon)$ of equation $(0.1)$ on the space $\mathbb{B}_{R(\varepsilon)}^{2}(\varepsilon)$, where

$$
\begin{aligned}
\mathbb{B}_{R}^{2}(\varepsilon):=\left\{(\theta, \xi) \in\left[H^{2}(\Omega) \cap H_{0}^{1}(\Omega)\right] \times \mathcal{E}^{1}(\varepsilon), \quad \xi=\right. & (u, v), \\
& \left.\mathbb{D}[u]+\|\theta\|_{H^{2}}+\|\xi\|_{\mathcal{E}^{1}(\varepsilon)} \leq R\right\}
\end{aligned}
$$

by the following standard expression:

$$
S_{t}(\varepsilon)\left(\theta_{0}, \xi^{0}\right):=\left(\theta(t), \xi_{u}(t)\right)
$$

where $\left(\theta(t), \xi_{u}(t)\right)$ is the unique solution of $(0.1)$ with initial data $\left(\theta_{0}, \xi^{0}\right)$ whose existence is proven in Theorem 2.1. Moreover, due to estimate (2.3), the set $\mathbb{B}_{R_{0}}(\varepsilon)$ is a uniform (with respect to $\varepsilon \leq \varepsilon_{0}$ ) absorbing set for (2.34), if $R_{0}$ is large enough, and there exists $T$ (which is independent of $\varepsilon$ ) such that

$$
S_{t}(\varepsilon): \mathbb{B}_{R_{0}}^{2}(\varepsilon) \rightarrow \mathbb{B}_{R_{0}}^{2}(\varepsilon), \quad \forall t \geq T,
$$

so that $\mathbb{B}_{R_{0}}^{2}(\varepsilon)$ is indeed an appropriate phase space for the study of the long-time behavior of solutions of problem (0.1). In the sequel, we will also need more regular solutions of problem (0.1). To this end, we introduce the space $\mathbb{B}_{R}^{3}(\varepsilon)$ as follows:

$$
\begin{array}{r}
\mathbb{B}_{R}^{3}(\varepsilon):=\left\{(\theta, \xi) \in\left[H^{3}(\Omega) \cap H_{0}^{1}(\Omega)\right] \times \mathcal{E}^{2}(\varepsilon), \xi=(u, v),\left.\Delta_{x} \theta\right|_{\partial \Omega}=0\right. \\
\left.-\left.\Delta_{x} u\right|_{\partial \Omega}=\left.g\right|_{\partial \Omega}, \quad \mathbb{D}[u]+\|\theta\|_{H^{3}}+\|\xi\|_{\mathcal{E}^{2}(\varepsilon)} \leq R\right\}
\end{array}
$$

Theorem 2.2. Let the assumptions of Theorem 2.1 hold. Then, for every $R>0$ and every initial data $\left(\theta(0), \xi_{u}(0)\right)$ belonging to $\mathbb{B}_{R(\varepsilon)}^{2}(\varepsilon) \cap \mathbb{B}_{R}^{3}(\varepsilon)$, the corresponding solution $\left(\theta(t), \xi_{u}(t)\right)$ of problem $(0.1)$ satisfies the following estimate

$$
\begin{aligned}
\|\theta(t)\|_{H^{3}}^{2} & +\left\|\partial_{t} \theta(t)\right\|_{H^{1}}^{2}+\left\|\xi_{u}(t)\right\|_{\mathcal{E}^{2}(\varepsilon)}^{2}+\int_{0}^{t} e^{-\alpha(t-s)}\left\|\partial_{t} u(s)\right\|_{H^{2}}^{2} d s \leq \\
& \leq Q\left(\mathbb{D}[u(0)]+\|\theta(0)\|_{H^{3}}^{2}+\left\|\xi_{u}(0)\right\|_{\mathcal{E}^{2}(\varepsilon)}^{2}\right) e^{-\alpha t}+Q\left(\|g\|_{H^{1} \cap L^{\infty}}\right),
\end{aligned}
$$

where the positive constant $\alpha$ and the monotonic function $Q$ are independent of $\varepsilon$.

Proof. We first deduce the required estimate for $\xi_{u}(t)$. To this end, we introduce the function $G=G(x) \in H^{3}(\Omega)$ as follows: $G:=\left(-\Delta_{x}\right)^{-1} g$. Then, the function $v(t):=u(t)-G$ solves the following hyperbolic problem:

$(2.38) \varepsilon \partial_{t}^{2} v+\partial_{t} v-\Delta_{x} v=\tilde{h}_{\theta, u}(t):=H^{\prime}(u(t)) \theta(t)-f(u(t)),\left.\quad v\right|_{\partial \Omega}=\left.\Delta_{x} v\right|_{\partial \Omega}=0$.

We have, thanks to estimate (2.3)

$$
\begin{aligned}
\left\|\tilde{h}_{\theta, u}(t)\right\|_{H^{2}}+\left\|\partial_{t} \tilde{h}_{\theta, u}(t)\right\|_{L^{2}} & \leq \\
& \leq Q\left(\mathbb{D}[u(0)]+\underset{25}{\left.\|\theta(0)\|_{H^{2}}+\left\|\xi_{u}(0)\right\|_{\mathcal{E}^{1}(\varepsilon)}\right) e^{-\alpha t}+Q\left(\|g\|_{L^{\infty}}\right)},\right.
\end{aligned}
$$


for appropriate positive constant $\alpha$ and monotonic function $Q$ which are independent of $\varepsilon$. Moreover, obviously, $\left.\tilde{h}_{\theta, u}(t)\right|_{\partial \Omega}=0$. Therefore, applying the $\mathcal{E}^{2}(\varepsilon)$-energy estimate (A.7) to the linear hyperbolic equation (2.38), we have

$$
\begin{aligned}
\left\|\xi_{v}(t)\right\|_{\mathcal{E}^{2}(\varepsilon)}^{2}+ & \int_{0}^{t} e^{-\alpha(t-s)}\left\|\partial_{t} v(s)\right\|_{H^{2}}^{2} d s \leq \\
& \leq C\left(\left\|\xi_{v}(0)\right\|_{\mathcal{E}^{2}(\varepsilon)}^{2}+\left\|\tilde{h}_{\theta, u}(0)\right\|_{H^{1}}^{2}\right) e^{-\alpha t}+ \\
& +C \int_{0}^{t} e^{-\alpha(t-s)}\left(\left\|\tilde{h}_{\theta, u}(s)\right\|_{H^{2}}^{2}+\left\|\partial_{t} h_{u, \theta}(s)\right\|_{L^{2}}^{2}\right) d s
\end{aligned}
$$

where the constants $C$ and $\alpha>0$ are independent of $\varepsilon$. Inserting estimate (2.39) into the right-hand side of $(2.40)$, we obtain the desired estimate for the $\mathcal{E}^{2}(\varepsilon)$-norm of $\xi_{u}(t)$.

In order to derive the desired estimates for the norms of $\theta(t)$, we rewrite the first equation of (0.1) in the form (1.9) and then apply the following regularity estimate:

$$
\begin{aligned}
\|\theta(t)\|_{H^{3}}^{2}+\int_{0}^{t} e^{-\alpha(t-s)}\left\|\partial_{t} \theta(s)\right\|_{H^{2}}^{2} d s \leq \\
\leq C\|\theta(0)\|_{H^{3}}^{2} e^{-\alpha t}+C \int_{0}^{t} e^{-\alpha(t-s)}\left\|h_{u}(s)\right\|_{H^{2}}^{2} d s
\end{aligned}
$$

(we recall that $\left.h_{u}(t)\right|_{\partial \Omega}=0$ ). Applying inequality (2.40) in order to estimate the last term in the right-hand side of (2.41), we deduce the desired estimates for $\|\theta(t)\|_{H^{3}}$ and $\int_{0}^{t} e^{-\alpha(t-s)}\left\|\partial_{t} \theta(s)\right\|_{H^{2}}^{2} d s$. The desired estimate for $\left\|\partial_{t} \theta(t)\right\|_{H^{1}}$ can be now obtained by expressing $\partial_{t} \theta(t)$ from the first equation of (0.1) and Theorem 2.2 is proven.

In the sequel, we also need the fact that $\mathbb{B}_{R}^{3}(\varepsilon)$ is a uniformly (with respect to $\varepsilon)$ exponentially attracting set for the semigroups $S_{t}(\varepsilon)$ for initial data belonging to $\mathbb{B}_{R(\varepsilon)}^{2}(\varepsilon)$.

Theorem 2.3. Let the assumptions of Theorem 2.2 hold. Then, there exist positive numbers $\bar{R}$ and $\alpha$ and a monotonic function $Q$ (which are independent of $\varepsilon$ ) such that

$$
\operatorname{dist}_{H^{2}(\Omega) \times \mathcal{E}^{1}(\Omega)}\left(S_{t}(\varepsilon) \mathbb{B}_{R}^{2}(\varepsilon), \mathbb{B}_{\bar{R}}^{3}(\varepsilon)\right) \leq Q(R) e^{-\alpha t}, \quad R \leq R(\varepsilon) .
$$

Proof. We first note that, due to estimate (2.3), it is sufficient to verify estimate $(2.42)$ for initial data belonging to $\mathbb{B}_{R_{0}}^{2}(\varepsilon)$ only (with sufficiently large, but fixed, $\left.R_{0}\right)$. To this end, we first split the solution $v$ of equation $(2.38)$ as follows: $v(t)=$ $v_{1}(t)+v_{2}(t)$, where $v_{1}(t)$ solves

$$
\varepsilon \partial_{t}^{2} v_{1}+\partial_{t} v_{1}-\Delta_{x} v_{1}=0, \quad \xi_{v_{1}}(0)=\xi_{v}(0),
$$


and the function $v_{2}(t)$ solves

$$
\varepsilon \partial_{t}^{2} v_{2}+\partial_{t} v_{2}-\Delta_{x} v_{2}=\tilde{h}_{\theta, u}(t), \quad \xi_{v_{2}}(0)=0 .
$$

Then, applying estimate (A.7) with $\kappa=1$ to equation (2.43), we have

$$
\left\|\xi_{v_{1}}(t)\right\|_{\mathcal{E}^{1}(\varepsilon)}^{2}+\int_{0}^{t} e^{-\alpha(t-s)}\left\|\partial_{t} v_{1}(s)\right\|_{H^{1}}^{2} d s \leq C e^{-\alpha t}\left\|\xi_{v}(0)\right\|_{\mathcal{E}^{1}(\varepsilon)}^{2},
$$

where the positive constants $C$ and $\alpha$ are independent of $\varepsilon$. Analogously, applying estimate (A.7) with $\kappa=2$ to equation (2.44) and using estimate (2.39), we obtain

$$
\left\|\xi_{v_{2}}(t)\right\|_{\mathcal{E}^{2}(\varepsilon)}^{2}+\int_{0}^{t} e^{-\alpha(t-s)}\left\|\partial_{t} v_{2}(s)\right\|_{H^{2}}^{2} d s \leq C_{R_{0}}
$$

where the constant $C_{R_{0}}$ is independent of $\varepsilon$ and $t$.

We now split the first component $\theta(t)$ as follows: $\theta(t)=\theta_{1}(t)+\theta_{2}(t)$, where $\theta_{1}$ solves

$$
\partial_{t} \theta_{1}-\Delta_{x} \theta_{1}=h_{u}^{1}(t):=-H^{\prime}(u(t)) \partial_{t} v_{1}(t), \quad \theta_{1}(0)=\theta(0),
$$

and the remainder $\theta_{2}(t)$ satisfies

$$
\partial_{t} \theta_{2}-\Delta_{x} \theta_{2}=h_{u}^{2}(t):=-H^{\prime}(u(t)) \partial_{t} v_{2}(t), \quad \theta_{2}(0)=0 .
$$

Applying the $H^{2}$-regularity estimate (1.18) (where $h_{u}$ is replaced by $h_{u}^{1}$ ) to the heat equation (2.47) and using estimates (2.45) and (2.3) in order to estimate the appropriate norm of $h_{u}^{1}(t)$, we have

$$
\left\|\theta_{1}(t)\right\|_{H^{2}}^{2} \leq C\left(\|\theta(0)\|_{H^{2}}^{2}+\left\|\xi_{u}(0)\right\|_{\mathcal{E}^{1}(\varepsilon)}^{2}\right) e^{-\alpha t},
$$

where the positive constants $C$ and $\alpha$ are independent of $t$. Moreover, applying the $H^{3}$-regularity estimate $(2.41)$ (where $h_{u}$ is replaced by $h_{u}^{2}$ ) and using estimates $(2.3)$ and (2.46) in order to estimate the integral of $\left\|h_{u}^{2}(t)\right\|_{H^{2}}$, we infer

$$
\left\|\theta_{2}(t)\right\|_{H^{3}} \leq C_{R_{0}}^{\prime}
$$

where the constant $C_{R_{0}}^{\prime}$ is independent of $t$. Estimates (2.45), (2.46), (2.49) and (2.50) give estimate (2.42) and finish the proof of Theorem 2.3.

Remark 2.1. We emphasize that Theorem 2.1 gives a unique solution of problem (0.1) only for initial data satisfying (2.2) (in particular, only for small $\varepsilon>0$ ). In fact, we cannot even construct a weak energy solution for this problem if this assumption is violated. Indeed, in contrast to the case of hyperbolic equations with regular nonlinearities and supercritical growth rate, see, e.g., [CV] and [Z], we now only have, a priori, in view of (2.6) (see also (A.4) in [MZ2]), an estimate for the nonlinear term $f(u)$ in $L^{1}([0, T] \times \Omega)$ (and not in the space $L^{1+\delta}([0, T] \times \Omega)$ with $\delta>0$ as in the case of regular nonlinearities) and, consequently, we do not know how to pass to the limit in the nonlinear term in a Galerkin approximation scheme and verify the existence of a weak solution.

Remark 2.2. In particular, we deduce from the results proved in this section the existence of strong solutions for weakly damped wave equations with singular potentials. This result is, to the best of our knowledge, the first one in this direction. 


\section{$\S 3$ Estimates on the Difference of SOlutions}

In this section, we derive several estimates on the difference of solutions of problem (0.1) which are of fundamental significance for our study of exponential attractors. We start with the following standard estimate.

Theorem 3.1. Let the assumptions of Theorem 2.1 hold. Then, for every $R \leq R(\varepsilon)$ and every solutions $\left(\theta_{1}(t), u_{1}(t)\right)$ and $\left(\theta_{2}(t), u_{2}(t)\right)$ of problem $(0.1)$ whose initial data belong to $\mathbb{B}_{R}^{2}(\varepsilon)$, the following estimate is valid

$$
\begin{aligned}
& (3.1) \quad\left\|\theta_{1}(t)-\theta_{2}(t)\right\|_{L^{2}}^{2}+\left\|\xi_{u_{1}}(t)-\xi_{u_{2}}(t)\right\|_{\mathcal{E}(\varepsilon)}^{2}+ \\
& +\int_{0}^{t}\left(\left\|\partial_{t} u_{1}(s)-\partial_{t} u_{2}(s)\right\|_{L^{2}}^{2}+\left\|\partial_{t} \theta_{1}(s)-\partial_{t} \theta_{2}(s)\right\|_{H^{-1}}^{2}+\left\|\theta_{1}(s)-\theta_{2}(s)\right\|_{H^{1}}^{2}\right) d s \leq \\
& \leq C e^{K t}\left(\left\|\theta_{1}(0)-\theta_{2}(0)\right\|_{L^{2}}^{2}+\left\|\xi_{u_{1}}(0)-\xi_{u_{2}}(0)\right\|_{\mathcal{E}(\varepsilon)}^{2}\right),
\end{aligned}
$$

where the constants $C$ and $K$ depend on $R$, but they are independent of $\varepsilon$.

Proof. We set $\bar{\theta}(t):=\theta_{1}(t)-\theta_{2}(t)$ and $\bar{u}(t):=u_{1}(t)-u_{2}(t)$. Then, these functions satisfy the following equations:

$$
\left\{\begin{array}{l}
\partial_{t} \bar{\theta}-\Delta_{x} \bar{\theta}=-\partial_{t}\left(H\left(u_{1}(t)\right)-H\left(u_{2}(t)\right)\right), \\
\varepsilon \partial_{t}^{2} \bar{u}+\partial_{t} \bar{u}-\Delta_{x} \bar{u}=h_{\bar{\theta}, \bar{u}}(t),
\end{array}\right.
$$

where

$$
h_{\bar{\theta}, \bar{u}}(t):=f\left(u_{2}(t)\right)-f\left(u_{1}(t)\right)+\left[H^{\prime}\left(u_{1}(t)\right)-H^{\prime}\left(u_{2}(t)\right)\right] \theta_{1}(t)-H^{\prime}\left(u_{2}(t)\right) \bar{\theta}(t) .
$$

We note that, thanks to estimate $(2.3)$,

$$
\left\|h_{\bar{\theta}, \bar{u}}(t)\right\|_{L^{2}} \leq C\left(\|\bar{u}(t)\|_{L^{2}}+\|\bar{\theta}(t)\|_{L^{2}}\right)
$$

where the constant $C$ depends on $R$, but it is independent of $\varepsilon$. The following lemma gives the estimate of $\partial_{t} h_{\bar{\theta}}, \bar{u}$ which is necessary for the study of the second equation of $(3.2)$.

Lemma 3.1. Let the above assumptions hold. Then

$$
\begin{aligned}
\left\|\partial_{t} h_{\bar{\theta}, \bar{u}}(t)\right\|_{H^{-1}} \leq C\left(1+\left\|\partial_{t} u_{2}(t)\right\|_{H^{1}}\right)\|\bar{\theta}(t)\|_{L^{2}}+ \\
+C\left(\left\|\partial_{t} \bar{\theta}(t)\right\|_{H^{-1}}+\|\bar{u}(t)\|_{H^{1}}+\left\|\partial_{t} \bar{u}(t)\right\|_{H^{-1}}\right) \\
+\quad
\end{aligned}
$$

where the constant $C$ is independent of $t$ and $\varepsilon$.

Proof. We have

$$
\begin{aligned}
& \partial_{t} h_{\bar{\theta}, \bar{u}}=\left[f^{\prime}\left(u_{2}\right)-f^{\prime}\left(u_{1}\right)\right] \partial_{t} u_{1}-f^{\prime}\left(u_{2}\right) \partial_{t} \bar{u}+\left[H^{\prime}\left(u_{1}\right)-H^{\prime}\left(u_{2}\right)\right] \partial_{t} \theta_{1}+ \\
& \quad+\theta_{1} H^{\prime \prime}\left(u_{2}\right) \partial_{t} \bar{u}+\partial_{t} u_{1}\left[H^{\prime \prime}\left(u_{1}\right)-H^{\prime \prime}\left(u_{2}\right)\right] \bar{\theta}-\partial_{t} u_{2} H^{\prime \prime}\left(u_{2}\right) \bar{\theta}-H^{\prime}\left(u_{2}\right) \partial_{t} \bar{\theta}
\end{aligned}
$$


We recall that, due to estimate (2.3) and the fact that the function $H$ is regular, we have $L^{\infty}$-bounds for $f^{(j)}\left(u_{i}\right)$ and $H^{(j)}\left(u_{i}\right)$. Consequently, since estimate $(2.3)$ gives a uniform estimate for $\left\|\partial_{t} u_{i}\right\|_{L^{2}}$ as well, then, for every $v \in H_{0}^{1}(\Omega)$, we have

$$
\begin{aligned}
\left|\left(\left[f^{\prime}\left(u_{2}\right)-f^{\prime}\left(u_{1}\right)\right] \partial_{t} u_{1}, v\right)_{L^{2}}\right| \leq\left\|f^{\prime}\left(u_{2}\right)-f^{\prime}\left(u_{1}\right)\right\|_{L^{3}}\left\|\partial_{t} u_{1}\right\|_{L^{2}}\|v\|_{L^{6}} \leq \\
\leq C\|\bar{u}\|_{L^{3}}\|v\|_{H^{1}}
\end{aligned}
$$

and, thus

$$
\left\|\left[f^{\prime}\left(u_{2}\right)-f^{\prime}\left(u_{1}\right)\right] \partial_{t} u_{1}\right\|_{H^{-1}} \leq C_{1}\|\bar{u}\|_{H^{1}}
$$

for some constants $C$ and $C_{1}$ which depend on $R$, but are independent of $\varepsilon$. Analogously, for the second term of (3.6), we have

$$
\begin{aligned}
& \left|\left(f^{\prime}\left(u_{2}\right) \partial_{t} \bar{u}, v\right)_{L^{2}}\right| \leq C\left\|\partial_{t} \bar{u}\right\|_{H^{-1}}\left\|f^{\prime}\left(u_{2}\right) v\right\|_{H^{1}} \leq \\
& \quad \leq C_{1}\left\|\partial_{t} \bar{u}\right\|_{H^{-1}}\left(\|v\|_{H^{1}}+\left\|\nabla_{x} u_{2} \cdot v\right\|_{L^{2}}\right) \leq C_{2}\left\|\partial_{t} \bar{u}\right\|_{H^{-1}}\|v\|_{H^{1}}\left(1+\left\|u_{2}\right\|_{H^{2}}\right)
\end{aligned}
$$

and, consequently, since $(2.3)$ gives an estimate for the $H^{2}$-norm of $u_{i}$,

$$
\left\|f^{\prime}\left(u_{2}\right) \partial_{t} \bar{u}\right\|_{H^{-1}} \leq C_{3}\left\|\partial_{t} \bar{u}\right\|_{H^{-1}} .
$$

The third term of (3.6) is completely analogous to the first one. Then, since, due to (2.3), we have an estimate for the $L^{2}$-norm of $\partial_{t} \theta_{1}$,

$$
\left\|\left[H^{\prime}\left(u_{1}\right)-H^{\prime}\left(u_{2}\right)\right] \partial_{t} \theta_{1}\right\|_{H^{-1}} \leq C\|\bar{u}\|_{H^{1}} .
$$

The fourth term of (3.6) is analogous to the second one and, consequently, analogously to (3.9) and (3.10), we have

$$
\begin{array}{r}
\left|\left(\theta_{1} H^{\prime \prime}\left(u_{2}\right) \partial_{t} \bar{u}, v\right)_{L^{2}}\right| \leq C_{1}\left\|\partial_{t} \bar{u}\right\|_{H^{-1}}\left\|\theta_{1} H^{\prime \prime}\left(u_{2}\right) v\right\|_{H^{1}} \leq \\
\leq C_{2}\left\|\partial_{t} \bar{u}\right\|_{H^{-1}}\left(\left\|\theta_{1}\right\|_{L^{\infty}}\|v\|_{H^{1}}+\left\|\left|\nabla_{x} \theta_{1}\right| \cdot|v|\right\|_{L^{2}}+\left\|\theta_{1}\right\|_{L^{\infty}}\left\|\left|\nabla_{x} u_{2}\right| \cdot|v|\right\|_{L^{2}}\right) \leq \\
\leq C_{3}\left\|\partial_{t} \bar{u}\right\|_{H^{-1}}\left(\left\|\theta_{1}\right\|_{H^{2}}^{2}+\left\|u_{2}\right\|_{H^{2}}^{2}\right)\|v\|_{H^{1}}
\end{array}
$$

and, therefore,

$$
\left\|\theta_{1} H^{\prime \prime}\left(u_{2}\right) \partial_{t} \bar{u}\right\|_{H^{-1}} \leq C\left\|\partial_{t} \bar{u}\right\|_{H^{-1}} .
$$

The fifth term of (3.6) is analogous to the third one. Consequently, due to Hölder's inequality with exponents $2,6,6$ and 6 , we have

$$
\begin{aligned}
& \left|\left(\partial_{t} u_{1}\left[H^{\prime \prime}\left(u_{1}\right)-H^{\prime \prime}\left(u_{2}\right)\right] \theta_{1}, v\right)_{L^{2}}\right| \leq \\
& \quad \leq C\left\|\partial_{t} u_{1}\right\|_{L^{2}}\left\|H^{\prime \prime}\left(u_{1}\right)-H^{\prime \prime}\left(u_{2}\right)\right\|_{L^{6}}\left\|\theta_{1}\right\|_{L^{6}}\|v\|_{L^{6}} \leq C_{1}\left\|u_{1}-u_{2}\right\|_{L^{6}}\|v\|_{H^{1}}
\end{aligned}
$$


and

$$
\left\|\partial_{t} u_{1}\left[H^{\prime \prime}\left(u_{1}\right)-H^{\prime \prime}\left(u_{2}\right)\right] \theta_{1}\right\|_{H^{-1}} \leq C_{3}\|\bar{u}\|_{H^{1}} .
$$

Analogously, applying Hölder's inequality to the sixth term of (3.6), we infer

$$
\begin{aligned}
\left|\left(\partial_{t} u_{2} H^{\prime \prime}\left(u_{2}\right) \bar{\theta}, v\right)_{L^{2}}\right| \leq C\left\|\partial_{t} u_{2}\right\|_{L^{4}}\left\|H^{\prime \prime}\left(u_{2}\right)\right\|_{L^{\infty}}\|\bar{\theta}\|_{L^{2}}\|v\|_{L^{4}} \leq \\
\leq C_{1}\left\|\partial_{t} u_{2}\right\|_{H^{1}}\|\bar{\theta}\|_{L^{2}}\|v\|_{H^{1}}
\end{aligned}
$$

and, consequently,

$$
\left\|\partial_{t} u_{2} H^{\prime \prime}\left(u_{2}\right) \bar{\theta}\right\|_{H^{-1}} \leq C_{2}\left\|\partial_{t} u_{2}\right\|_{H^{1}}\|\bar{\theta}\|_{L^{2}}
$$

Combining estimates (3.8), (3.10) and (3.12-3.14), we deduce (3.5) and finish the proof of Lemma 3.1.

Thus, applying the $\mathcal{E}(\varepsilon)$-energy estimate (A.2) to the second equation of (3.2), using (3.4) and (3.5) and estimate (2.3) for the integral of the $H^{1}$-norm of $\partial_{t} u_{2}$, we have

$$
\begin{aligned}
& \left\|\xi_{\bar{u}}(t)\right\|_{\mathcal{E}(\varepsilon)}^{2}+\int_{0}^{t} e^{-\alpha(t-s)}\left\|\partial_{t} \bar{u}(s)\right\|_{L^{2}}^{2} d s \leq \\
& \leq C_{1}\left(\left\|\xi_{\bar{u}}(0)\right\|_{\mathcal{E}(\varepsilon)}^{2}+\left\|h_{\bar{\theta}, \bar{u}}(0)\right\|_{H^{-1}}^{2}\right) e^{-\alpha t}+ \\
& +C_{1} \int_{0}^{t} e^{-\alpha(t-s)}\left(\left\|h_{\bar{\theta}, \bar{u}}(s)\right\|_{L^{2}}^{2}+\left\|\partial_{t} h_{\bar{\theta}, \bar{u}}(s)\right\|_{H^{-1}}^{2}\right) d s \leq C_{2}\left\|\xi_{\bar{u}}(0)\right\|_{\mathcal{E}(\varepsilon)}^{2}+ \\
& +C_{2} \int_{0}^{t}\left(\|\bar{u}(s)\|_{H^{1}}^{2}+\left\|\partial_{t} \bar{u}(s)\right\|_{H^{-1}}^{2}+\left\|\partial_{t} \bar{\theta}(s)\right\|_{H^{-1}}^{2}\right) d s+C_{2} \sup _{s \in[0, t]}\|\bar{\theta}(s)\|_{L^{2}}^{2},
\end{aligned}
$$

where the constants $C_{1}$ and $C_{2}$ are independent of $\varepsilon$. Multiplying now the first equation of $(3.2)$ by $\left(-\Delta_{x}\right)^{-1} \partial_{t} \bar{\theta}(t)$ and integrating over $[0, t] \times \Omega$, we have

$$
\begin{aligned}
\int_{0}^{t}\left\|\partial_{t} \bar{\theta}(s)\right\|_{H^{-1}}^{2} d s+\|\bar{\theta}(t)\|_{L^{2}}^{2} & \leq\|\bar{\theta}(0)\|_{L^{2}}^{2}+ \\
& +C \int_{0}^{t}\left\|\partial_{t}\left(H\left(u_{1}(s)\right)-H\left(u_{2}(s)\right)\right)\right\|_{H^{-1}}^{2} d s .
\end{aligned}
$$

Moreover, noting that $\partial_{t}\left[H\left(u_{1}\right)-H\left(u_{2}\right)\right]=H^{\prime}\left(u_{1}\right) \partial_{t} \bar{u}+\left[H^{\prime}\left(u_{1}\right)-H^{\prime}\left(u_{2}\right)\right] \partial_{t} u_{2}$ and arguing as in the proof of Lemma 3.1, we can verify that

$$
\left\|\partial_{t}\left[H\left(u_{1}(s)\right)-H\left(u_{2}(s)\right)\right]\right\|_{H^{-1}} \leq C\left(\left\|\partial_{t} \bar{u}(s)\right\|_{H^{-1}}+\|\bar{u}(s)\|_{H^{1}}\right)
$$

(see (3.7-3.10)). Inserting this estimate into the right-hand side of (3.16), we have

$$
\begin{aligned}
\sup _{s \in[0, t]}\|\bar{\theta}(s)\|_{L^{2}}^{2}+ & \int_{0}^{t}\left\|\partial_{t} \bar{\theta}(s)\right\|_{H^{-1}} d s \leq \\
& \leq C\|\bar{\theta}(0)\|_{L^{2}}^{2}+C \int_{0}^{t}\left(\left\|\partial_{t} \bar{u}(s)\right\|_{H^{-1}}^{2}+\|\bar{u}(s)\|_{H^{1}}^{2}\right) d s .
\end{aligned}
$$


Inserting estimate (3.18) into the right-hand side of (3.15), we infer

$$
\begin{aligned}
& \left\|\xi_{\bar{u}}(t)\right\|_{\mathcal{E}(\varepsilon)}^{2}+\int_{0}^{t} e^{-\alpha(t-s)}\left\|\partial_{t} \bar{u}(s)\right\|_{L^{2}}^{2} d s \leq \\
& \quad \leq C\left(\|\bar{\theta}(0)\|_{L^{2}}^{2}+\left\|\xi_{\bar{u}}(0)\right\|_{\mathcal{E}(\varepsilon)}^{2}\right)+C \int_{0}^{t}\left(\left\|\partial_{t} \bar{u}(s)\right\|_{H^{-1}}^{2}+\|\bar{u}(s)\|_{H^{1}}^{2}\right) d s .
\end{aligned}
$$

Applying Gronwall's inequality, we find

$$
\left\|\xi_{\bar{u}}(t)\right\|_{\mathcal{E}(\varepsilon)}^{2}+\int_{0}^{t}\left\|\partial_{t} \bar{u}(s)\right\|_{L^{2}}^{2} d s \leq C_{1} e^{K t}\left(\|\bar{\theta}(0)\|_{L^{2}}^{2}+\left\|\xi_{\bar{u}}(0)\right\|_{\mathcal{E}(\varepsilon)}^{2}\right)
$$

for appropriate constants $C_{1}$ and $K$ which depend on $R$, but are independent of $\varepsilon$ and $t$. Thus, the $\bar{u}$-part of estimate (3.1) is proven. In order to obtain the desired estimates for $\|\bar{\theta}(t)\|_{L^{2}}$ and $\int_{0}^{t}\left\|\partial_{t} \bar{\theta}(s)\right\|_{H^{-1}}^{2} d s$, it is sufficient to insert estimate (3.20) into the right-hand side of (3.18). Thus, it only remains to estimate $\int_{0}^{t}\|\bar{\theta}(s)\|_{H^{1}}^{2} d s$. To this end, we note that, expressing $\Delta_{x} \bar{\theta}(t)$ from the first equation of (3.2) and taking the $H^{-1}$-norm of both sides of the equation that we obtain, we have

$$
\|\bar{\theta}(s)\|_{H^{1}}^{2} \leq\left\|\partial_{t} \bar{\theta}(s)\right\|_{H^{-1}}^{2}+\left\|\partial_{t}\left(H\left(u_{1}(s)\right)-H\left(u_{2}(s)\right)\right)\right\|_{H^{-1}}^{2} .
$$

Integrating (3.21) over $[0, t]$ and using estimates (3.17), (3.18) and (3.20), we deduce the desired estimate for the integral of $\|\bar{\theta}(s)\|_{H^{1}}$. This finishes the proof of Theorem 3.1 .

We now derive an asymptotic smoothing property for the difference of solutions of problem (0.1). To this end, we split the solution $(\bar{\theta}(t), \bar{u}(t))$ of problem $(3.2)$ as follows:

$$
(\bar{\theta}(t), \bar{u}(t))=\left(\bar{\theta}_{1}(t), \bar{u}_{1}(t)\right)+\left(\bar{\theta}_{2}(t), \bar{u}_{2}(t)\right)
$$

where $\left(\bar{\theta}_{1}(t), \bar{u}_{1}(t)\right)$ solves

$$
\left\{\begin{array}{l}
\partial_{t} \bar{\theta}_{1}-\Delta_{x} \bar{\theta}_{1}=0,\left.\quad \bar{\theta}_{1}\right|_{t=0}=\left.\bar{\theta}\right|_{t=0}, \\
\varepsilon \partial_{t}^{2} \bar{u}_{1}+\partial_{t} \bar{u}_{1}-\Delta_{x} \bar{u}_{1}=0,\left.\quad \xi_{\bar{u}_{1}}\right|_{t=0}=\left.\xi_{\bar{u}}\right|_{t=0},
\end{array}\right.
$$

and the rest $\left(\bar{\theta}_{2}(t), \bar{u}_{2}(t)\right)$ solves

$$
\left\{\begin{array}{l}
\partial_{t} \bar{\theta}_{2}-\Delta_{x} \bar{\theta}_{2}=-\partial_{t}\left(H\left(u_{1}(t)\right)-H\left(u_{2}(t)\right)\right),\left.\quad \bar{\theta}_{2}\right|_{t=0}=0 \\
\varepsilon \partial_{t}^{2} \bar{u}_{2}+\partial_{t} \bar{u}_{2}-\Delta_{x} \bar{u}_{2}=h_{\bar{\theta}, \bar{u}}(t),\left.\quad \xi_{\bar{u}_{1}}\right|_{t=0}=0 .
\end{array}\right.
$$


Theorem 3.2. Let the assumptions of Theorem 2.1 be satisfied and let $\left(\theta_{1}(t), u_{1}(t)\right)$ and $\left(\theta_{2}(t), u_{2}(t)\right)$ be two solutions of problem $(0.1)$ with initial data in $\mathbb{B}_{R}^{2}(\varepsilon)$, for some $R \leq R(\varepsilon)$. Then, the solutions $\left(\bar{\theta}_{1}(t), \bar{u}_{1}(t)\right)$ and $\left(\bar{\theta}_{2}(t), \bar{u}_{2}(t)\right)$ satisfy the following estimates

$$
\left\{\begin{array}{l}
\left\|\bar{\theta}_{1}(t)\right\|_{L^{2}}^{2}+\left\|\xi_{\bar{u}_{1}}(t)\right\|_{\mathcal{E}(\varepsilon)}^{2} \leq C e^{-\alpha t}\left(\|\bar{\theta}(0)\|_{L^{2}}^{2}+\left\|\xi_{\bar{u}}(0)\right\|_{\mathcal{E}(\varepsilon)}^{2}\right) \\
\left\|\bar{\theta}_{2}(t)\right\|_{H^{1}}^{2}+\left\|\xi_{\bar{u}_{2}}(t)\right\|_{\mathcal{E}^{1}(\varepsilon)}^{2} \leq C e^{K t}\left(\|\bar{\theta}(0)\|_{L^{2}}^{2}+\left\|\xi_{\bar{u}}(0)\right\|_{\mathcal{E}(\varepsilon)}^{2}\right)
\end{array}\right.
$$

where the positive constants $C, K, \alpha$ depend on $R$, but they are independent of $\varepsilon$.

Proof. Since system (3.23) consists of two decoupled linear parabolic and hyperbolic equations, the first estimate of (3.25) is obvious and we only need to prove the second one. To this end, we note that

$$
\left\|h_{\bar{\theta}, \bar{u}}(t)\right\|_{H^{1}} \leq C\left(\|\bar{u}(t)\|_{H^{1}}+\|\bar{\theta}(t)\|_{H^{1}}\right),
$$

where the constant $C$ depends on $R$, but it is independent of $\varepsilon$. Indeed, estimate (3.26) can be verified as in the proof of Lemma 3.1 and even in a simpler way, since we now do not need to estimate the negative norms (to this end, it is sufficient to estimate the $L^{2}$-norm of $\nabla_{x} h_{\bar{\theta}, \bar{u}}(t)$ and, in order to do so, we only need to estimate the $L^{2}$-norm of every term in the right-hand side of (3.6), where the operator $\partial_{t}$ is replaced by $\nabla_{x}$ ). We thus leave the details to the reader.

Recalling now that $\left.h_{\bar{\theta}, \bar{u}}\right|_{\partial \Omega}=0$, applying the $\mathcal{E}^{1}(\varepsilon)$-energy estimate (A.7) to the second equation of (3.24) and using estimates (3.1), (3.5) and (3.26), we have

$$
\begin{gathered}
\left\|\xi_{\bar{u}_{2}}(t)\right\|_{\mathcal{E}^{1}(\varepsilon)}^{2} \leq C_{1} \int_{0}^{t}\left(\left\|h_{\bar{\theta}, \bar{u}}(s)\right\|_{H^{1}}^{2}+\left\|\partial_{t} h_{\bar{\theta}, \bar{u}}(s)\right\|_{H^{-1}}^{2}\right) d s \leq \\
\leq C_{2} \int_{0}^{t}\left[\left(1+\left\|\partial_{t} u_{2}(s)\right\|_{H^{1}}^{2}\right)\|\bar{\theta}(s)\|_{L^{2}}^{2}+\|\bar{\theta}(s)\|_{H^{1}}^{2}+\left\|\partial_{t} \bar{\theta}(s)\right\|_{H^{-1}}^{2}+\left\|\partial_{t} \bar{\theta}(s)\right\|_{H^{-1}}^{2}\right] d s \leq \\
\leq C_{3} e^{K t}\left(\|\bar{\theta}(0)\|_{L^{2}}^{2}+\left\|\xi_{\bar{u}}(0)\right\|_{\mathcal{E}(\varepsilon)}^{2}\right),
\end{gathered}
$$

where the constants $C_{i}$ and $K$ are independent of $\varepsilon$. Thus, the $\bar{u}_{2}$-part of the second estimate of (3.25) is obtained. In order to obtain the $\bar{\theta}_{2}$-part, it remains to note that, due to estimates (2.3) and (3.1),

$$
\begin{gathered}
\int_{0}^{t}\left\|\partial_{t}\left[H\left(u_{1}(s)\right)-H\left(u_{2}(s)\right)\right]\right\|_{L^{2}}^{2} d s \leq \\
\leq \int_{0}^{t}\left(\left\|H^{\prime}\left(u_{1}(s)\right) \partial_{t} \bar{u}(s)\right\|_{L^{2}}^{2}+\left\|\left(H^{\prime}\left(u_{1}(s)\right)-H^{\prime}\left(u_{2}(s)\right)\right) \partial_{t} u_{2}(s)\right\|_{L^{2}}^{2}\right) d s \leq \\
\quad \leq C \int_{0}^{t}\left\|\partial_{t} \bar{u}(s)\right\|_{L^{2}}^{2} d s+C \int_{0}^{t}\left\|\partial_{t} u_{2}(s)\right\|_{H^{1}}^{2}\|\bar{u}(s)\|_{H^{1}}^{2} d s \leq \\
\quad \leq C_{4} e^{K t}\left(\|\bar{\theta}(0)\|_{L^{2}}^{2}+\left\|\xi_{\bar{u}}(0)\right\|_{\mathcal{E}(\varepsilon)}^{2}\right)
\end{gathered}
$$


and apply the classical $L^{2}$-regularity estimate (see $\left(1.9^{\prime}\right)$ ) to the first equation of (3.24). This finishes the proof of Theorem 3.2.

We now estimate the difference between the solutions $\left(\theta^{\varepsilon}(t), u^{\varepsilon}(t)\right)$ of problem (0.1) for small positive $\varepsilon$ and the solutions $\left(\theta^{0}(t), u^{0}(t)\right)$ of the limit parabolic problem (2.1). To this end, we need the first term of the asymptotic expansion of $\left(\theta^{\varepsilon}, u^{\varepsilon}\right)$ near $t=0$ with respect to $\varepsilon$. Following the general scheme (see $[\mathrm{LyV}]$ ), we seek for asymptotic expansions of the form

$$
\theta^{\varepsilon}(t)=\theta^{0}(t)+\varepsilon \mathcal{P}(t), \quad u^{\varepsilon}(t)=u^{0}(t)+\varepsilon \tilde{u}^{1}\left(\varepsilon^{-1} t\right)+\varepsilon \mathcal{R}(t),
$$

where $\left(\theta^{0}, u^{0}\right)$ solves the limit parabolic problem (1.1) with initial data $\left(\theta^{0}(0), u^{0}(0)\right)$ $=\left(\theta^{\varepsilon}(0), u^{\varepsilon}(0)\right)$, the boundary layer term $\tilde{u}^{1}(\tau)$ satisfies the following equation:

$$
\partial_{\tau}^{2} \tilde{u}^{1}+\partial_{\tau} \tilde{u}^{1}=0, \quad \partial_{\tau} \tilde{u}^{1}(0)=\partial_{t} u^{0}(0)-\partial_{t} u^{\varepsilon}(0) \text { and } \lim _{\tau \rightarrow+\infty} \tilde{u}^{1}(\tau)=0,
$$

and $(\mathcal{P}(t), \mathcal{R}(t))$ is the remainder. Solving (3.28), we have

$$
\tilde{u}^{1}(\tau)=e^{-\tau} \phi_{\theta, u}^{\varepsilon}(0),
$$

where

$$
\phi_{\theta, u}^{\varepsilon}(t):=\varepsilon \partial_{t}^{2} u^{\varepsilon}(t)=g-\partial_{t} u^{\varepsilon}(t)+\Delta_{x} u^{\varepsilon}(t)-f\left(u^{\varepsilon}(t)\right)+H^{\prime}\left(u^{\varepsilon}(t)\right) \theta^{\varepsilon}(t) .
$$

The next theorem gives an estimate of the remainder $(\mathcal{P}, \mathcal{R})$ in expansions (3.27).

Theorem 3.3. Let $\left(\theta^{\varepsilon}(t), u^{\varepsilon}(t)\right)$ be a solution of problem (0.1) with initial data belonging to $\mathbb{B}_{R(\varepsilon)}^{2}(\varepsilon) \cap \mathbb{B}_{R}^{3}(\varepsilon)$ for some $R>0$ and let the external forces $g$ belong to the space $L^{\infty}(\Omega) \cap H^{1}(\Omega)$. Then, the remainder $(\mathcal{P}(t), \mathcal{R}(t))$ in the asymptotic expansions (3.27) satisfies the following estimate

$$
\|\mathcal{P}(t)\|_{L^{2}}^{2}+\left\|\xi_{\mathcal{R}}(t)\right\|_{\mathcal{E}(\varepsilon)}^{2} \leq C e^{K t}, \quad \xi_{\mathcal{R}}(t):=\left(\mathcal{R}(t), \partial_{t} \mathcal{R}(t)\right),
$$

where the constants $C$ and $K$ depend on $R$, but they are independent of $\varepsilon$.

Proof. It is not difficult to verify that the remainder $(\mathcal{P}(t), \mathcal{R}(t))$ satisfies the following equations:

$$
\left\{\begin{array}{l}
\partial_{t} \mathcal{P}-\Delta_{x} \mathcal{P}=\partial_{t} \frac{1}{\varepsilon}\left[H\left(u^{0}(t)\right)-H\left(u^{0}(t)+\varepsilon \tilde{u}^{1}\left(\varepsilon^{-1} t\right)+\varepsilon \mathcal{R}(t)\right)\right],\left.\mathcal{P}\right|_{t=0}=0 \\
\varepsilon \partial_{t}^{2} \mathcal{R}+\partial_{t} \mathcal{R}-\Delta_{x} \mathcal{R}=h_{\mathcal{P}, \mathcal{R}}(t)-\partial_{t}^{2} u^{0}(t),\left.\quad \xi_{\mathcal{R}}\right|_{t=0}=\left(-\phi_{\theta, u}(0), 0\right)
\end{array}\right.
$$

where $\left(\theta^{0}(t), u^{0}(t)\right)$ is the corresponding solution of the limit parabolic problem $(2.1)$ and

$$
\begin{aligned}
& \text { 3.33) } h_{\mathcal{P}, \mathcal{R}}(t):=\frac{1}{\varepsilon}\left[f\left(u^{0}(t)\right)-f\left(u^{\varepsilon}(t)\right)\right]+ \\
& +\frac{1}{\varepsilon}\left[H^{\prime}\left(u^{\varepsilon}(t)\right) \theta^{\varepsilon}(t)-H^{\prime}\left(u^{0}(t)\right) \theta^{0}(t)\right]+\Delta_{x} \tilde{u}^{1}\left(\varepsilon^{-1} t\right):=\frac{1}{\varepsilon} \tilde{h}_{\mathcal{P}, \mathcal{R}}(t)+\Delta_{x} \tilde{u}^{1}\left(\varepsilon^{-1} t\right) .
\end{aligned}
$$


We split the function $\mathcal{R}(t)$ as follows: $\mathcal{R}(t)=\mathcal{R}_{1}(t)+\mathcal{R}_{2}(t)$, where

$$
\begin{array}{r}
\varepsilon \partial_{t}^{2} \mathcal{R}_{1}+\partial_{t} \mathcal{R}_{1}-\Delta_{x} \mathcal{R}_{1}=-\partial_{t}^{2} u^{0}(t),\left.\quad \xi_{\mathcal{R}_{1}}\right|_{t=0}=0, \\
\varepsilon \partial_{t}^{2} \mathcal{R}_{2}+\partial_{t} \mathcal{R}_{2}-\Delta_{x} \mathcal{R}_{2}=h_{\mathcal{P}, \mathcal{R}}(t),\left.\quad \xi_{\mathcal{R}_{2}}\right|_{t=0}=\left(-\phi_{\theta, u}(0), 0\right) .
\end{array}
$$

Applying Proposition A.1 to the first equation of (3.34) and using estimates (1.67), we have

$$
\left\|\xi_{\mathcal{R}_{1}}(t)\right\|_{\mathcal{E}(\varepsilon)}^{2} \leq C
$$

where the constant $C$ depends on $R$, but it is independent of $t$ and $\varepsilon$. Analogously, applying Proposition A.2 to the second equation of (3.34), we deduce that

$$
\begin{aligned}
\left\|\xi_{\mathcal{R}_{2}}(t)\right\|_{\mathcal{E}(\varepsilon)}^{2} \leq & C e^{-\alpha t}+ \\
& +C\left(\int_{0}^{t} e^{-\alpha(t-s)}\left(\left\|h_{\mathcal{P}, \mathcal{R}}(s)\right\|_{H^{-1}}+\left\|\partial_{t} h_{\mathcal{P}, \mathcal{R}}(s)\right\|_{H^{-1}}\right) d s\right)^{2} .
\end{aligned}
$$

Thus, we need to estimate the right-hand side of (3.36). To this end, we note that the function $\tilde{h}_{\mathcal{P}, \mathcal{R}}(t)$ coincides with the function $(3.3)$, where now $u_{1}(t):=u^{0}(t)$, $\theta_{1}(t):=\theta^{0}(t), u_{1}(t):=u^{0}(t)$ and $u_{2}(t):=u^{\varepsilon}(t)$. Consequently, using estimates $(3.4)$ and (3.5) and the fact that, thanks to estimates (1.66) and (2.37), the $H^{1}$-norm of $\partial_{t} u^{\varepsilon}(t)$ is also uniformly bounded, we infer

$$
\begin{gathered}
\left\|\tilde{h}_{\mathcal{P}, \mathcal{R}}(t)\right\|_{H^{-1}}+\left\|\partial_{t} \tilde{h}_{\mathcal{P}, \mathcal{R}}(t)\right\|_{H^{-1}} \leq \\
\leq C\left(\left\|\theta^{0}(t)-\theta^{\varepsilon}(t)\right\|_{L^{2}}+\left\|u^{0}(t)-u^{\varepsilon}(t)\right\|_{H^{1}}+\right. \\
\left.+\left\|\partial_{t} \theta^{0}(t)-\partial_{t} \theta^{\varepsilon}(t)\right\|_{H^{-1}}+\left\|\partial_{t} u^{0}(t)-\partial_{t} u^{\varepsilon}(t)\right\|_{H^{-1}}\right) \leq \\
\leq C_{1} \varepsilon\left(\|\mathcal{P}(t)\|_{L^{2}}+\|\mathcal{R}(t)\|_{H^{1}}+\right. \\
\left.+\left\|\partial_{t} \mathcal{P}(t)\right\|_{H^{-1}}+\left\|\partial_{t} \mathcal{R}(t)\right\|_{H^{-1}}\right)+C_{1} \varepsilon\left(\left\|\tilde{u}^{1}\left(\varepsilon^{-1} t\right)\right\|_{H^{1}}+\left\|\partial_{t} \tilde{u}^{1}\left(\varepsilon^{-1} t\right)\right\|_{H^{-1}}\right),
\end{gathered}
$$

where the constants $C$ and $C_{1}$ depend on $R$, but they are independent of $\varepsilon$ and $t$. We also note that, thanks to (3.29), (3.30) and Theorem 2.2, we have

$$
\left\|\tilde{u}^{1}\left(\varepsilon^{-1} t\right)\right\|_{H^{1}} \leq C e^{-t / \varepsilon}, \quad\left\|\partial_{t} \tilde{u}^{1}\left(\varepsilon^{-1} t\right)\right\|_{H^{-1}} \leq C \varepsilon^{-1} e^{-t / \varepsilon} .
$$

Moreover, expressing $\partial_{t} \mathcal{P}(t)$ from the first equation of (3.32), taking the $H^{-1}$-norm of both sides of the equation that we obtain and using (3.17), we have

$$
\begin{gathered}
\text { (3.39) }\left\|\partial_{t} \mathcal{P}(t)\right\|_{H^{-1}} \leq\|\mathcal{P}(t)\|_{H^{1}}+\left\|\frac{1}{\varepsilon} \partial_{t}\left[H\left(u^{0}(t)\right)-H\left(u^{\varepsilon}(t)\right)\right]\right\|_{H^{-1}} \leq \\
\leq\|\mathcal{P}(t)\|_{H^{1}}+C \varepsilon^{-1}\left(\left\|u^{0}(t)-u^{\varepsilon}(t)\right\|_{H^{1}}+\left\|\partial_{t} u^{0}(t)-\partial_{t} u^{\varepsilon}(t)\right\|_{H^{-1}}\right) \leq \\
\leq\|\mathcal{P}(t)\|_{H^{1}}+C\left(\|\mathcal{R}(t)\|_{H^{1}}+\left\|\partial_{t} \mathcal{R}(t)\right\|_{H^{-1}}\right)+C\left(\left\|\tilde{u}^{1}\left(\varepsilon^{-1} t\right)\right\|_{H^{1}}+\left\|\partial_{t} \tilde{u}^{1}\left(\varepsilon^{-1} t\right)\right\|_{H^{-1}}\right) .
\end{gathered}
$$


Estimates (3.37-3.39) imply that

$$
\begin{aligned}
\left\|h_{\mathcal{P}, \mathcal{R}}(t)\right\|_{H^{-1}} & +\left\|\partial_{t} h_{\mathcal{P}, \mathcal{R}}(t)\right\|_{H^{-1}} \leq \\
& \leq C\left(\|\mathcal{R}(t)\|_{H^{1}}+\left\|\partial_{t} \mathcal{R}(t)\right\|_{H^{-1}}+\|\mathcal{P}(t)\|_{H^{1}}+\varepsilon^{-1} e^{-\varepsilon^{-1} t}\right)
\end{aligned}
$$

where the constant $C$ depends on $R$, but it is independent of $t$ and $\varepsilon$. Inserting this estimate into the right-hand side of (3.36) and using (3.35), we have

$$
\begin{array}{r}
+C_{2}\left(\int_{0}^{t} e^{-\alpha(t-s)}\left(\left\|\partial_{t} \mathcal{R}(s)\right\|_{H^{-1}}+\|\mathcal{P}(s)\|_{H^{1}}+\|\mathcal{R}(s)\|_{H^{1}}+\varepsilon^{-1} e^{-s / \varepsilon}\right) d s\right)^{2} \leq \\
\leq C_{3}+C_{4} \int_{0}^{t}\left(\|\mathcal{R}(s)\|_{H^{1}}^{2}+\left\|\partial_{t} \mathcal{R}(s)\right\|_{H^{-1}}^{2}+\|\mathcal{P}(s)\|_{H^{1}}^{2}\right) d s
\end{array}
$$

where the constants $C_{i}$ and $\alpha$ are independent of $\varepsilon$ and $t$. Thus, we only need to estimate the integral of the $H^{1}$-norm of $\mathcal{P}(t)$. To this end, we first note that, analogously to the proof of Lemma 3.1,

$$
\begin{aligned}
& \left\|\frac{1}{\varepsilon}\left[H\left(u^{\varepsilon}(t)\right)-H\left(u^{0}(t)\right)\right]\right\|_{H^{1}} \leq C \varepsilon^{-1}\left\|u^{0}(t)-u^{\varepsilon}(t)\right\|_{H^{1}} \leq \\
& \leq C\|\mathcal{R}(t)\|_{H^{1}}+C\left\|\tilde{u}^{1}\left(\varepsilon^{-1} t\right)\right\|_{H^{1}} \leq C_{1}\left(1+\|\mathcal{R}(t)\|_{H^{1}}\right) .
\end{aligned}
$$

Consequently, multiplying the first equation of (3.32) by $\mathcal{P}(t)+\frac{1}{\varepsilon}\left[H\left(u^{\varepsilon}(t)\right)-\right.$ $\left.H\left(u^{0}(t)\right)\right]$, integrating over $[0, t] \times \Omega$ and arguing analogously to $(1.29)$, we have

$$
\begin{aligned}
& \| \mathcal{P}(t)+\frac{1}{\varepsilon}[\left.H\left(u^{\varepsilon}(t)\right)-H\left(u^{0}(t)\right)\right]\left\|_{L^{2}}^{2}+\int_{0}^{t}\right\| \mathcal{P}(s) \|_{H^{1}}^{2} d s \leq \\
& \leq C_{1}\left\|\mathcal{P}(0)+\frac{1}{\varepsilon}\left[H\left(u^{\varepsilon}(0)\right)-H\left(u^{0}(0)\right)\right]\right\|_{L^{2}}^{2}+ \\
&+C_{1} \int_{0}^{t}\left\|\frac{1}{\varepsilon}\left[H\left(u^{\varepsilon}(t)\right)-H\left(u^{0}(t)\right)\right]\right\|_{H^{1}}^{2} d s \leq \\
& \leq C_{2}(t+1)+C_{2} \int_{0}^{t}\|\mathcal{R}(s)\|_{H^{1}}^{2} d s,
\end{aligned}
$$

where the constants $C_{i}$ are independent of $\varepsilon$. Inserting the estimate of the integral of $\|\mathcal{P}(s)\|_{H^{1}}^{2}$ obtained in (3.43) into the right-hand side of (3.41) and recalling the definition of the $\mathcal{E}(\varepsilon)$-norm, we finally have

$$
\left\|\xi_{\mathcal{R}}(t)\right\|_{\mathcal{E}(\varepsilon)}^{2} \leq C\left(t+1+\int_{0}^{t}\left\|\xi_{\mathcal{R}}(s)\right\|_{\mathcal{E}(\varepsilon)}^{2} d s\right) .
$$


Applying Gronwall's inequality to (3.44), we deduce that

$$
\left\|\xi_{\mathcal{R}}(t)\right\|_{\mathcal{E}(\varepsilon)} \leq C^{\prime} e^{K t}
$$

where the positive constants $C^{\prime}$ and $K$ depend on $R$, but they are independent of $t$ and $\varepsilon$. Thus, the $\mathcal{R}$-part of estimate (3.31) is proven. The desired estimate for the $L^{2}$-norm of $\mathcal{P}(t)$ now follows from (3.42), (3.43) and (3.45), which finishes the proof of Theorem 3.3.

Corollary 3.1. Let the assumptions of Theorem 3.3 hold. Then, the following estimate is valid

$$
\begin{aligned}
& \left\|\xi_{u^{\varepsilon}}(t)-\xi_{u^{0}}(t)\right\|_{\mathcal{E}(\varepsilon)}+\left\|\theta^{\varepsilon}(t)-\theta^{0}(t)\right\|_{L^{2}} \leq \\
& \quad \leq C \varepsilon e^{K t}+C e^{-\varepsilon^{-1} t}\left(\left\|\phi_{\theta, u}^{\varepsilon}(0)\right\|_{H^{-1}}+\varepsilon^{1 / 2}\left\|\phi_{\theta, u}(0)\right\|_{L^{2}}\right),
\end{aligned}
$$

where the constants $C$ and $K$ depend on $R$, but they are independent of $\varepsilon$.

Indeed, (3.46) follows from the asymptotic expansions (3.27) and Theorem 3.3.

We now generalize estimate (3.46) to the case where the solutions $\left(\theta^{\varepsilon}, u^{\varepsilon}\right)$ and $\left(\theta^{0}, u^{0}\right)$ have different initial data.

Corollary 3.2. Let the assumptions of Theorem 3.3 hold and let $(\theta(t), u(t))$ be a solution of the limit parabolic problem (1.1) with initial data in $\mathbb{B}_{R}^{3}(0)$. Then, the following estimate is valid

$$
\begin{aligned}
\left\|\xi_{u^{\varepsilon}}(t)-\xi_{u}(t)\right\|_{\mathcal{E}(0)} & \leq C e^{K t}\left(\left\|\theta^{\varepsilon}(0)-\theta(0)\right\|_{L^{2}}+\left\|u^{\varepsilon}(0)-u(0)\right\|_{H^{1}}\right) \\
& +C \varepsilon e^{K t}+C e^{\varepsilon^{-1} t}\left(\left\|\phi_{\theta, u}^{\varepsilon}(0)\right\|_{H^{-1}}+\varepsilon^{1 / 2}\left\|\phi_{\theta, u}^{\varepsilon}(0)\right\|_{L^{2}}\right),
\end{aligned}
$$

where the constants $C$ and $K$ depend on $R$, but they are independent of $\varepsilon$.

Indeed, let $\left(\theta^{0}(t), u^{0}(t)\right)$ be the same as in Theorem 3.3. Then, on the one hand, the difference $u^{\varepsilon}(t)-u^{0}(t)$ satisfies estimate (3.46) and, on the other hand, since $\left(\theta^{0}(t), u^{0}(t)\right)$ and $(\theta(t), u(t))$ solve the limit parabolic equation (1.1), then, thanks to estimate (3.1) with $\varepsilon=0$, we have

$$
\begin{aligned}
& \left\|\theta(t)-\theta^{0}(t)\right\|_{L^{2}}^{2}+\left\|\xi_{u}(t)-\xi_{u^{0}}(t)\right\|_{\mathcal{E}(0)}^{2} \leq \\
& \leq C e^{K t}\left(\left\|\theta(0)-\theta^{0}(0)\right\|_{L^{2}}^{2}+\left\|\xi_{u}(0)-\xi_{u^{0}}(0)\right\|_{\mathcal{E}(0)}^{2}\right) \leq \\
& \quad \leq C_{1} e^{K t}\left(\left\|\theta(0)-\theta^{0}(0)\right\|_{L^{2}}^{2}+\left\|u(0)-u^{0}(0)\right\|_{H^{1}}^{2}\right) .
\end{aligned}
$$

Combining (3.46) and (3.48), we deduce (3.47).

In the sequel, we need to control the evolution of quantity (3.30). 
Corollary 3.3. Let the assumptions of Theorem 3.3 hold. Then, the following estimate is valid

$$
\left\|\phi_{\theta, u}^{\varepsilon}(t)\right\|_{H^{-1}}+\varepsilon^{1 / 2}\left\|\phi_{\theta, u}^{\varepsilon}(t)\right\|_{L^{2}} \leq C\left(e^{\varepsilon^{-1} t}\left\|\phi_{\theta, u}^{\varepsilon}(0)\right\|_{H^{-1}}+\varepsilon\right),
$$

where the constant $C$ is independent of $\varepsilon$ and $t$.

Proof. Inserting the asymptotic expansions (3.27) into (3.30), we have

$$
\begin{gathered}
(3.50) \quad \phi_{\theta, u}^{\varepsilon}(t)=\varepsilon \Delta_{x} \tilde{u}^{1}\left(\varepsilon^{-1} t\right)-\partial_{\tau} \tilde{u}^{1}\left(\varepsilon^{-1} t\right)+\varepsilon \Delta_{x} \mathcal{R}(t)-\varepsilon \partial_{t} \mathcal{R}(t)+ \\
+\left[f\left(u^{\varepsilon}(t)\right)-f\left(u^{0}(t)\right)\right]+ \\
+\left[H^{\prime}\left(u^{\varepsilon}(t)\right) \theta^{\varepsilon}(t)-H^{\prime}\left(u^{0}(t)\right) \theta^{0}(t)\right]=\varepsilon h_{\mathcal{P}, \mathcal{R}}(t)-\partial_{\tau} \tilde{u}^{1}\left(\varepsilon^{-1} t\right)+\varepsilon \Delta_{x} \mathcal{R}(t)-\varepsilon \partial_{t} \mathcal{R}(t),
\end{gathered}
$$

where $h_{\mathcal{P}, \mathcal{R}}(t)$ is defined by (3.33). Moreover, without loss of generality, we can assume that $t \leq 1$. Then, using estimates (3.4), (3.31), (3.33) and (3.38), we have

$$
\begin{aligned}
& \left\|\phi_{\theta, u}^{\varepsilon}(t)\right\|_{H^{-1}} \leq C \varepsilon\left(\|\mathcal{R}(t)\|_{H^{1}}+\left\|\partial_{t} \mathcal{R}(t)\right\|_{H^{-1}}+\|\mathcal{P}(t)\|_{L^{2}}\right)+ \\
& +C \varepsilon\left(\left\|\tilde{u}^{1}\left(\varepsilon^{-1} t\right)\right\|_{H^{1}}+\left\|\partial_{t} \tilde{u}^{1}\left(\varepsilon^{-1} t\right)\right\|_{H^{-1}}\right) \leq \\
& \quad \leq C\left(e^{-\varepsilon^{-1} t}\left\|\phi_{\theta, u}^{\varepsilon}(0)\right\|_{H^{-1}}+\varepsilon\right)
\end{aligned}
$$

where the constant $C$ is independent of $\varepsilon$ and $t \in[0,1]$. Moreover, it follows from (1.66) and (2.37) that

$$
\left\|\phi_{\theta, u}^{\varepsilon}(t)\right\|_{H^{1}} \leq C_{1}
$$

Finally, interpolating between $H^{-1}$ and $H^{1}$, we derive the desired estimate for the quantity $\varepsilon^{1 / 2}\left\|\phi_{\theta, u}^{\varepsilon}(t)\right\|_{L^{2}}$ and finish the proof of Corollary 3.3.

Remark 3.1. We recall that, according to (3.30), estimate (3.49) can be rewritten as follows:

$$
\left\|\partial_{t}^{2} u^{\varepsilon}(t)\right\|_{H^{-1}}+\varepsilon^{1 / 2}\left\|\partial_{t}^{2} u^{\varepsilon}(t)\right\|_{L^{2}} \leq C_{1} \frac{1}{\varepsilon} e^{-\varepsilon^{-1} t}+C_{2}
$$

where the constants $C_{1}$ and $C_{2}$ depend on $R$, but they are independent of $\varepsilon$.

\section{$\S 4$ Robust Exponential ATtRACTORS}

In this section, we construct a robust family of exponential attractors $\mathcal{M}_{\varepsilon}$ for problems (0.1) as $\varepsilon \rightarrow 0$. To this end, we first note that the semigroups (2.35) generated by equation (0.1) with $\varepsilon>0$ and the semigroup (1.60) associated with the limit parabolic problem (1.1) are defined on different phase spaces (since the parabolic problem (1.1) does not require to have an initial data for the derivative $\left.\partial_{t} u\right|_{t=0}$ ). In order to overcome this difficulty, following the standard procedure (for the theory of 
singularly perturbed hyperbolic equations, see, e.g., [BV] and [FGMZ]), we define the infinite dimensional submanifold $\mathcal{N}_{0}$ of the space $L^{2}(\Omega) \times \mathcal{E}(0)$ as follows:

$$
\begin{aligned}
\mathcal{N}_{0}:=\left\{(\theta,[u, v]) \in H^{2}(\Omega) \times \mathcal{E}^{1}(0),\right. & \mathbb{D}[u(0)]+\|\theta\|_{H^{2}}+\|u\|_{H^{2}}<\infty \\
v & \left.=\mathcal{N}(\theta, u):=g+\Delta_{x} u-f(u)+H^{\prime}(u) \theta\right\}
\end{aligned}
$$

and define the semigroup $S_{t}(0): \mathcal{N}_{0} \rightarrow \mathcal{N}_{0}$ associated with the limit parabolic equation (1.1) via

$$
S_{t}(0)\left(\theta_{0}, u_{0}, v_{0}\right):=\left(S_{t}\left(\theta_{0}, u_{0}\right), \mathcal{N}\left(S_{t}\left(\theta_{0}, u_{0}\right)\right)\right), \quad\left(\theta_{0}, u_{0}, v_{0}\right) \in \mathcal{N}_{0} .
$$

The main result of this section is the following theorem.

Theorem 4.1. Let the assumptions of Theorem 2.1 hold and let the external forces $g$ in $(0.1)$ belong to $L^{\infty}(\Omega) \cap H^{1}(\Omega)$. Then, there exists a positive number $R_{0}$ and a family of exponential attractors $\mathcal{M}_{\varepsilon}, \varepsilon \in\left[0, \varepsilon_{0}\right]$, of the semigroups $S_{t}(\varepsilon)$ enjoying the following properties

1) the following inclusions hold

$$
\mathcal{M}_{\varepsilon} \subset \mathbb{B}_{R_{0}}^{3}(\varepsilon), \quad S_{t}(\varepsilon) \mathcal{M}_{\varepsilon} \subset \mathcal{M}_{\varepsilon}, \quad \forall t \in \mathbb{R}_{+}, \varepsilon \in\left[0, \varepsilon_{0}\right] ;
$$

2) the fractal dimension of $\mathcal{M}_{\varepsilon}$ is uniformly bounded with respect to $\varepsilon$

$$
\operatorname{dim}_{F}\left(\mathcal{M}_{\varepsilon}, H^{2}(\Omega) \times \mathcal{E}^{1}(\varepsilon)\right) \leq C, \quad \varepsilon \in\left[0, \varepsilon_{0}\right] ;
$$

3) the attractors $\mathcal{M}_{\varepsilon}$ converge to the limit attractor $\mathcal{M}_{0}$ in the following sense

$$
\operatorname{dist}_{H^{2}(\Omega) \times \mathcal{E}^{1}(\varepsilon)}^{\text {sym }}\left(\mathcal{M}_{\varepsilon}, \mathcal{M}_{0}\right) \leq C \varepsilon^{\kappa}
$$

where dist $_{V}^{\text {sym }}$ denotes the symmetric distance between sets in the space $V$ and the constants $C$ and $K$ are independent of $\varepsilon$;

4) the sets $\mathcal{M}_{\varepsilon}$ attract exponentially the trajectories of the semigroups $S_{t}(\varepsilon)$, i.e., there exist a positive constant $\alpha$ and a monotonic function $Q$ (which are independent of $\varepsilon$ ) such that, for every $\varepsilon \leq \varepsilon_{0}$ and $R \leq R(\varepsilon)$,

$$
\operatorname{dist}_{H^{2}(\Omega) \times \mathcal{E}^{1}(\varepsilon)}\left(S_{t}(\varepsilon) \mathbb{B}_{R}^{2}(\varepsilon), \mathcal{M}_{\varepsilon}\right) \leq Q(R) e^{-\alpha t}, \quad \forall t \in \mathbb{R}_{+} .
$$

Proof. We first construct the exponential attractors $\mathcal{M}_{\varepsilon}$ for more regular initial data (i.e., belonging to $\mathbb{B}_{R(\varepsilon)}^{2}(\varepsilon) \cap \mathbb{B}_{R}^{3}(\varepsilon)$ ). Thanks to estimate (2.3) and (2.37), it is sufficient to construct the exponential attractors $\mathcal{M}_{\varepsilon}$ for initial data belonging to the set $\mathcal{B}_{\varepsilon}:=\mathbb{B}_{R_{0}}^{3}(\varepsilon)$ only (where $R_{0} \geq 2 Q\left(\|g\|_{L^{\infty} \cap H^{1}}\right.$ ) is large enough). Moreover, according to these estimates, there exists $T=T\left(R_{0}\right)$, which is independent of $\varepsilon \in$ $\left(0, \varepsilon_{0}\right)$, such that

$$
S_{t}(\varepsilon) \mathcal{B}_{\varepsilon} \subset \mathcal{B}_{\varepsilon}, \quad \text { for all } t \geq T
$$


For the limit case $\varepsilon=0$, it is however more convenient to define the set $\mathcal{B}_{0}$ in a slightly different way, namely

$$
\mathcal{B}_{0}:=\left\{(\theta,[u, v]) \in \mathcal{N}_{0}, \mathbb{D}[u]+\|\theta\|_{H^{3}}+\|u\|_{H^{3}} \leq R_{0}\right\}
$$

Then, on the one hand, obviously, $\mathcal{B}_{0} \subset \mathbb{B}_{\bar{R}}^{3}(0)$, where $\bar{R}=\bar{R}\left(R_{0}\right)$ is large enough, and, on the other hand, due to estimate (1.64), $\mathcal{B}_{0}$ is an absorbing set for the semigroup $S_{t}(0)$ if $R_{0}$ is large enough and, moreover, due to estimate (1.66), we have

$$
S_{t} \mathcal{B}_{0} \subset \mathcal{B}_{0}, \quad \text { for all } t \geq T
$$

Thus, instead of constructing the exponential attractors $\mathcal{M}_{\varepsilon}$ for the continuous semigroups $S_{t}(\varepsilon)$, we first construct the exponential attractors $\mathcal{M}_{\varepsilon}^{d}$ for the discrete semigroups $S_{\varepsilon}^{(n)}:=S_{n T}(\varepsilon)$ acting on the phase spaces $\mathcal{B}_{\varepsilon}$ :

$$
S_{\varepsilon}^{(n)}: \mathcal{B}_{\varepsilon} \rightarrow \mathcal{B}_{\varepsilon}, \quad \forall n \in \mathbb{N}, \quad \varepsilon \in\left[0, \varepsilon_{0}\right]
$$

To this end, we apply the abstract theorem on perturbations of exponential attractors proven in [FGMZ]. According to this theorem, we need to verify that there exist two families of Banach spaces $E(\varepsilon)$ and $E^{1}(\varepsilon), \varepsilon \in\left[0, \varepsilon_{0}\right]$, such that:

1 ) the set $\mathcal{B}_{\varepsilon}$ is a closed bounded subset of $E(\varepsilon), \mathcal{B}_{0} \subset E(\varepsilon)$ for all $\varepsilon \in\left[0, \varepsilon_{0}\right]$ and

$$
\left\|b_{0}\right\|_{E(\varepsilon)} \leq C_{1}\left\|b_{0}\right\|_{E(0)}+C_{2} \varepsilon^{\delta}, \forall b_{0} \in \mathcal{B}_{0}
$$

where the positive constants $C_{1}, C_{2}$ and $\delta$ are independent of $\varepsilon$;

2 ) the space $E^{1}(\varepsilon)$ is compactly embedded into the space $E(\varepsilon), \forall \varepsilon \in\left[0, \varepsilon_{0}\right]$, and this compactness is uniform with respect to $\varepsilon$ in the following sense:

$$
N_{\mu}\left(B\left(1,0, E^{1}(\varepsilon)\right), E(\varepsilon)\right) \leq \mathbb{M}(\mu), \quad \forall \mu>0
$$

where $B\left(1,0, E^{1}(\varepsilon)\right)$ is the unit ball of $E^{1}(\varepsilon), N_{\mu}(X, V)$ denotes the minimal number of $\mu$-balls in $V$ which are necessary to cover the subset $X \subset V$ and the monotonic decreasing function $\mathbb{M}$ is independent of $\varepsilon$;

3) there exist two maps $\mathcal{C}_{\varepsilon}$ and $\mathcal{K}_{\varepsilon}$ (which map $\mathcal{B}_{\varepsilon}$ onto $E(\varepsilon)$ ) such that $S_{\varepsilon}:=$ $S_{\varepsilon}^{(1)}=\mathcal{C}_{\varepsilon}+\mathcal{K}_{\varepsilon}$ and, for every $b_{\varepsilon}^{1}, b_{\varepsilon}^{2} \in \mathcal{B}_{\varepsilon}$, we have

$$
\left\{\begin{array}{l}
\left\|\mathcal{K}_{\varepsilon} b_{\varepsilon}^{1}-\mathcal{K}_{\varepsilon} b_{\varepsilon}^{2}\right\|_{E^{1}(\varepsilon)} \leq K\left\|b_{\varepsilon}^{1}-b_{\varepsilon}^{2}\right\|_{E(\varepsilon)} \\
\left\|\mathcal{C}_{\varepsilon} b_{\varepsilon}^{1}-\mathcal{C}_{\varepsilon} b_{\varepsilon}^{2}\right\|_{E(\varepsilon)} \leq \delta\left\|b_{\varepsilon}^{1}-b_{\varepsilon}^{2}\right\|_{E(\varepsilon)}
\end{array}\right.
$$

where $\delta<1 / 2$ and $K$ are independent of $\varepsilon$;

4) there exist nonlinear projectors $\Pi_{\varepsilon}: \mathcal{B}_{\varepsilon} \rightarrow \mathcal{B}_{0}$ such that $\Pi_{\varepsilon} \mathcal{B}_{\varepsilon}=\mathcal{B}_{0}$ and

$$
\left\|S_{\varepsilon}^{(n)} b_{\varepsilon}-S_{0}^{(n)} \Pi_{\varepsilon} b_{\varepsilon}\right\|_{E(\varepsilon)} \underset{39}{\leq} C \varepsilon L^{n}, \quad n \in \mathbb{N}, \quad b_{\varepsilon} \in \mathcal{B}_{\varepsilon}
$$


where the constants $C$ and $L$ are also independent of $\varepsilon$.

Let us verify these conditions for the semigroups (4.9) generated by problems (0.1). To this end, we set

$$
E^{i}(\varepsilon):=H^{i}(\Omega) \times \mathcal{E}^{i}(\varepsilon), \quad i=0,1 .
$$

Then, the first condition (with $\delta=1 / 2$ in (4.10)) follows immediately from the definition of the sets $\mathcal{B}_{\varepsilon}$. The second assumption is also obvious for the spaces $E(\varepsilon):=E^{0}(\varepsilon)$. The third assumption follows from Theorem 3.2 if $T$ is large enough (we recall that $S_{\varepsilon}:=S_{T}(\varepsilon)$ ). So, it only remains to verify the fourth assumption. To this end, we define the projectors $\Pi_{\varepsilon}:=\Pi$ by the following obvious expression:

$$
\Pi(\theta,[u, v]):=(\theta,[u, \mathcal{N}(\theta, u)])
$$

where the map $\mathcal{N}$ is the same as in (4.1). Then, it follows from the definition of the set $\mathcal{B}_{0}$ that $\Pi \mathcal{B}_{\varepsilon} \subset \mathcal{B}_{0}$. Moreover, since $(\theta,[u, 0]) \in \Pi^{-1}(\theta,[u, \mathcal{N}(u, \theta)])$, then, $\Pi \mathcal{B}_{\varepsilon}=\mathcal{B}_{0}$, for every $\varepsilon>0$. It remains to note that estimate (4.13) is an immediate consequence of estimate (3.46) (see also (3.49)). Thus, all the assumptions of the abstract theorem on the existence of a robust family of exponential attractors are verified and, consequently, due to this theorem (see [FGMZ]), there exists a family $\mathcal{M}_{\varepsilon}^{d} \subset \mathcal{B}_{\varepsilon}$ of exponential attractors for the semigroups $S_{\varepsilon}^{(n)}$ such that:

$$
\text { 1) } \operatorname{dist}_{E(\varepsilon)}\left(S_{\varepsilon}^{(n)} \mathcal{B}_{\varepsilon}, \mathcal{M}_{\varepsilon}^{d}\right) \leq C e^{-L n}
$$

where the constants $C$ and $L$ are independent of $t$ and $\varepsilon$,

$$
\text { 2) } \operatorname{dim}_{F}\left(\mathcal{M}_{\varepsilon}^{d}, E(\varepsilon)\right) \leq C_{1} \text {, }
$$

where $C_{1}$ is independent of $\varepsilon$, and

$$
\text { 3) } \operatorname{dist}_{E(\varepsilon)}^{\text {sym }}\left(\mathcal{M}_{\varepsilon}^{d}, \mathcal{M}_{0}^{d}\right) \leq C_{2} \varepsilon^{\kappa}
$$

where the positive constants $C_{2}$ and $\kappa$ are independent of $\varepsilon$.

Thus, the desired exponential attractors for the discrete semigroups are constructed. In order to obtain the exponential attractors for the continuous semigroups $S_{t}(\varepsilon)$, we use the following standard formula:

$$
\mathcal{M}_{\varepsilon}:=\cup_{t \in[T, 2 T]} S_{t}(\varepsilon) \mathcal{M}_{\varepsilon}^{d} .
$$

Let us verify that the attractors $\mathcal{M}_{\varepsilon}$ so constructed satisfy all the assumptions of Theorem 4.1. Indeed, it can be proven, using estimates (2.37), (3.1) and (3.52), that the semigroups $S_{t}(\varepsilon)$ are uniformly Lipschitz continuous on $[T, 2 T] \times \mathcal{B}_{\varepsilon}$ in the metric of $E(\varepsilon)$ (see [FGMZ]). Consequently, due to (4.16) and (4.17), we have:

$$
\left\{\begin{array}{l}
\operatorname{dist}_{E(\varepsilon)}\left(S_{t}(\varepsilon) \mathcal{B}_{\varepsilon}, \mathcal{M}_{\varepsilon}\right) \leq C^{\prime} e^{-L^{\prime} t}, \\
\operatorname{dim}_{F}\left(\mathcal{M}_{\varepsilon}, E(\varepsilon)\right) \leq C_{1}^{\prime},
\end{array}\right.
$$


where the new constants $C^{\prime}, C_{1}^{\prime}$ and $L^{\prime}$ are independent of $\varepsilon$. Moreover, using now estimates (3.47), (3.49) and (4.10), we derive the analogue of (4.18) for the continuous attractors

$$
\operatorname{dist}_{E(\varepsilon)}^{\text {sym }}\left(\mathcal{M}_{\varepsilon}, \mathcal{M}_{0}\right) \leq C_{2}^{\prime} \varepsilon^{\kappa},
$$

see [FGMZ] for the details. We also recall that, due to Theorem 2.2, the trajectories of the semigroups $S_{t}(\varepsilon)$ are uniformly bounded in $H^{3}(\Omega) \times \mathcal{E}^{2}(\varepsilon)$ and, consequently, using an appropriate interpolation inequality, we deduce that estimates (4.20) and (4.21) remain valid with the spaces $E(\varepsilon)$ replaced by $H^{2}(\Omega) \times \mathcal{E}^{1}(\varepsilon)$ (of course, with different constants $C^{\prime}, C_{1}^{\prime}, C_{2}^{\prime}, L^{\prime}$ and $\kappa$ which are independent of $\varepsilon$ ).

Thus, all the assertions of Theorem 4.1, except (4.5), are satisfied and instead of estimate (4.5), we now only have

$$
\operatorname{dist}_{H^{1}(\Omega) \times \mathcal{E}^{1}(\varepsilon)}\left(S_{t}(\varepsilon)\left[\mathbb{B}_{R(\varepsilon)}^{2}(\varepsilon) \cap \mathbb{B}_{R}^{3}(\varepsilon)\right], \mathcal{M}_{\varepsilon}\right) \leq Q(R) e^{-\alpha t},
$$

where the positive constant $\alpha$ and the monotonic function $Q$ are independent of $\varepsilon$ (here, we have implicitly used the fact that $\mathcal{B}_{\varepsilon}$ is an absorbing set for the semigroups $S_{t}(\varepsilon)$ with initial data belonging to $\mathbb{B}_{R(\varepsilon)}^{2} \cap\left[H^{3}(\Omega) \times \mathcal{E}^{2}(\varepsilon)\right]$ ). Using now estimate (4.22), Theorem 2.3 and the transitivity of exponential attraction (see [FGMZ]), we derive estimate (4.5) for initial data belonging to $\mathbb{B}_{R}^{2}(\varepsilon)$ and finish the proof of Theorem 4.1.

Remark 4.1. It follows from Theorem 4.1 that there exists a family of global attractors $\mathcal{A}_{\varepsilon}, \varepsilon \in\left[0, \varepsilon_{0}\right]$, of the semigroups $S_{t}(\varepsilon)$ enjoying the following properties 1) $\mathcal{A}_{\varepsilon} \subset \mathbb{B}_{R_{0}}^{3}(\varepsilon), \quad S_{t}(\varepsilon) \mathcal{A}_{\varepsilon}=\mathcal{A}_{\varepsilon}, \quad \forall t \in \mathbb{R}_{+}, \varepsilon \in\left[0, \varepsilon_{0}\right]$

2) the fractal dimension of $\mathcal{A}_{\varepsilon}$ is uniformly bounded with respect to $\varepsilon$, that is,

$$
\begin{gathered}
\operatorname{dim}_{F}\left(\mathcal{A}_{\varepsilon}, H^{2}(\Omega) \times \mathcal{E}^{1}(\varepsilon)\right) \leq C, \quad \varepsilon \in\left[0, \varepsilon_{0}\right] . \\
\text { APPENDIX: UNIFORM ENERGY ESTIMATES } \\
\text { FOR THE LINEAR HYPERBOLIC PROBLEM }
\end{gathered}
$$

Here, we give several uniform (with respect to $\varepsilon \rightarrow 0$ ) energy estimates for the following initial and boundary value problem for a singularly perturbed damped hyperbolic equation:

$$
\varepsilon \partial_{t}^{2} v+\partial_{t} v-\Delta_{x} v=h(t),\left.\quad \xi_{v}\right|_{t=0}=\xi^{0},\left.\quad v\right|_{\partial \Omega}=0
$$

which are necessary in order to handle the second equation of (0.1). We start with the following result.

Proposition A.1. Let $v$ be a solution of (A.1). Then, the following estimate is valid

$$
\begin{aligned}
\left\|\xi_{v}(t)\right\|_{\mathcal{E}(\varepsilon)}^{2}+\int_{0}^{t} e^{-\alpha(t-s)}\left\|\partial_{t} v(s)\right\|_{L^{2}}^{2} d s \leq \\
\leq C e^{-\alpha t}\left(\left\|\xi_{v}(0)\right\|_{\mathcal{E}(\varepsilon)}^{2}+\|h(0)\|_{H^{-1}}^{2}\right)+ \\
\quad+C \int_{0}^{t} e^{-\alpha(t-s)}\left(\|h(s)\|_{L^{2}}^{2}+\left\|\left(-\Delta_{x}\right)^{-1} \partial_{t} h(s)\right\|_{L^{2}}^{2}\right) d s
\end{aligned}
$$


where the positive constants $\alpha$ and $C$ are independent of $\varepsilon \rightarrow 0$.

Proof. Multiplying equation (A.1) by $\partial_{t} v(t)+\alpha v(t)$, where $\alpha>0$ is a sufficiently small (but independent of $\varepsilon$ ) number, integrating over $\Omega$ and arguing in a standard way, we have

$$
\begin{aligned}
& \varepsilon\left\|\partial_{t} v(t)\right\|_{L^{2}}^{2}+\|v(t)\|_{H^{1}}^{2}+\int_{0}^{t} e^{-\alpha(t-s)}\left\|\partial_{t} v(s)\right\|_{L^{2}}^{2} d s \leq \\
& \leq C e^{-\alpha t}\left(\varepsilon\left\|\partial_{t} v(0)\right\|_{L^{2}}^{2}+\|v(0)\|_{H^{1}}^{2}\right)+C \int_{0}^{t} e^{-\alpha(t-s)}\|h(s)\|_{L^{2}}^{2} d s
\end{aligned}
$$

where the constant $C$ is independent of $\varepsilon$ (see, e.g., [BV]). Thus, it only remains to deduce the estimate for the $H^{-1}$-norm of $\partial_{t} v$. To this end, we multiply equation (A.1) by $\left(-\Delta_{x}\right)^{-1} \partial_{t}^{2} v(t)$ and integrate over $\Omega$. Then, we have

$$
\begin{gathered}
\varepsilon\left\|\partial_{t}^{2} v(t)\right\|_{H^{-1}}^{2}+\frac{d}{d t}\left[\frac{1}{2}\left\|\partial_{t} v(t)\right\|_{H^{-1}}^{2}+\left(v(t), \partial_{t} v(t)\right)_{L^{2}}-\right. \\
\left.-\left(h(t),\left(-\Delta_{x}\right)^{-1} \partial_{t} v(t)\right)_{L^{2}}\right]+\alpha\left[\frac{1}{2}\left\|\partial_{t} v(t)\right\|_{H^{-1}}^{2}+\left(v(t), \partial_{t} v(t)\right)_{L^{2}}-\right. \\
\left.-\left(h(t),\left(-\Delta_{x}\right)^{-1} \partial_{t} v(t)\right)_{L^{2}}\right]=-\left(\left(-\Delta_{x}\right)^{-1} \partial_{t} h(t), \partial_{t} v(t)\right)_{L^{2}} \\
-\left\|\partial_{t} v(t)\right\|_{L^{2}}^{2}+\alpha\left[\frac{1}{2}\left\|\partial_{t} v(t)\right\|_{H^{-1}}^{2}+\left(v(t), \partial_{t} v(t)\right)_{L^{2}}-\left(h(t),\left(-\Delta_{x}\right)^{-1} \partial_{t} v(t)\right)_{L^{2}}\right] .
\end{gathered}
$$

Applying now Gronwall's inequality to (A.4) and using (A.3) in order to estimate the right-hand side of (A.4), we have

$$
\begin{aligned}
& \frac{1}{2}\left\|\partial_{t} v(t)\right\|_{H^{-1}}^{2}+\left(v(t), \partial_{t} v(t)\right)_{L^{2}}-\left(h(t),\left(-\Delta_{x}\right)^{-1} \partial_{t} v(t)\right)_{L^{2}} \leq \\
& \leq\left[\frac{1}{2}\left\|\partial_{t} v(0)\right\|_{H^{-1}}^{2}+\left(v(0), \partial_{t} v(0)\right)_{L^{2}}-\left(h(0),\left(-\Delta_{x}\right)^{-1} \partial_{t} v(0)\right)_{L^{2}}\right] e^{-\alpha t}+ \\
& \quad+C \int_{0}^{t} e^{-\alpha(t-s)}\left(\|h(s)\|_{L^{2}}^{2}+\left\|\left(-\Delta_{x}\right)^{-1} \partial_{t} h(s)\right\|_{L^{2}}^{2}\right) d s,
\end{aligned}
$$

where the constant $C$ is independent of $\varepsilon$. We now recall that, for every $\alpha>0$,

$$
\begin{gathered}
\|h(t)\|_{H^{-1}}^{2}-e^{-\alpha t}\|h(0)\|_{H^{-1}}^{2}=\int_{0}^{t} \frac{d}{d s}\left(e^{-\alpha(t-s)}\|h(s)\|_{H^{-1}}^{2}\right) d s= \\
=\int_{0}^{t} e^{-\alpha(t-s)}\left(\alpha\|h(s)\|_{H^{-1}}^{2}+2\left(h(s),\left(-\Delta_{x}\right)^{-1} \partial_{t} h(s)\right)_{L^{2}} d s \leq\right. \\
\leq C_{\alpha} \int_{0}^{t} e^{-\alpha(t-s)}\left(\|h(s)\|_{L^{2}}^{2}+\left\|\left(-\Delta_{x}\right)^{-1} \partial_{t} h(s)\right\|_{L^{2}}^{2}\right) d s .
\end{gathered}
$$

Estimate (A.5), together with (A.3) and (A.6), imply that

$$
\begin{aligned}
\left\|\partial_{t} v(t)\right\|_{H^{-1}}^{2} \leq C\left(\left\|\xi_{v}(0)\right\|_{\mathcal{E}(\varepsilon)}^{2}\right. & \left.+\|h(0)\|_{H^{-1}}^{2}\right) e^{-\alpha t}+ \\
& +C \int_{0}^{t} e^{-\alpha(t-s)}\left(\|h(s)\|_{L^{2}}^{2}+\left\|\left(-\Delta_{x}\right)^{-1} \partial_{t} h(s)\right\|_{L^{2}}^{2}\right) d s,
\end{aligned}
$$

which finishes the proof of Proposition A.1. 
Corollary A.1. Let $v$ be a solution of problem (A.1) and let us assume, in addition, that $\left.h(t)\right|_{\partial \Omega}=0$ for all $t \geq 0$. Then, the following estimate is valid

$$
\begin{aligned}
& \left\|\xi_{v}(t)\right\|_{\mathcal{E}^{\kappa}(\varepsilon)}^{2}+\int_{0}^{t} e^{-\alpha(t-s)}\left\|\partial_{t} v(s)\right\|_{H^{\kappa}}^{2} d s \leq \\
& \leq C e^{-\alpha t}\left(\left\|\xi_{v}(0)\right\|_{\mathcal{E}^{\kappa}(\varepsilon)}^{2}+\|h(0)\|_{H^{\kappa-1}}^{2}\right)+ \\
& \quad+\int_{0}^{t} e^{-\alpha(t-s)}\left(\|h(s)\|_{H^{\kappa}}^{2}+\left\|\partial_{t} h(s)\right\|_{H^{\kappa-2}}^{2}\right) d s,
\end{aligned}
$$

where $\kappa=1,2$ and the positive constants $\alpha$ and $C$ are independent of $\varepsilon$.

Indeed, applying the operator $\left(-\Delta_{x}\right)^{\kappa / 2}$ to both sides of equation (A.1) and applying estimate (A.2) to the equation that we obtain, we deduce estimate (A.7).

The following proposition gives the analogue of estimate (A.2) in the case where the $W^{1,1}\left([0, T], H^{-1}(\Omega)\right)$-norm of the right-hand side $h$ is known.

Proposition A.2. Let $v$ be a solution of problem (A.1). Then, the following estimate is valid

$$
\begin{aligned}
& \left\|\xi_{v}(t)\right\|_{\mathcal{E}(\varepsilon)}^{2}+\int_{0}^{t} e^{-\alpha(t-s)}\left\|\partial_{t} v(s)\right\|_{L^{2}}^{2} d s \leq \\
& \leq C e^{-\alpha t}\left(\left\|\xi_{v}(0)\right\|_{\mathcal{E}(\varepsilon)}^{2}+\|h(0)\|_{H^{-1}}^{2}\right)+ \\
& \quad+C\left(\int_{0}^{t} e^{-\alpha(t-s)}\left(\left\|\partial_{t} h(s)\right\|_{H^{-1}}+\|h(s)\|_{H^{-1}}\right) d s\right)^{2},
\end{aligned}
$$

where the positive constants $\alpha$ and $C$ are independent of $\varepsilon$.

Proof. Multiplying equation (A.1) by $2\left(\partial_{t} v(t)+\alpha v(t)\right)$, where $\alpha>0$ is a small (but independent of $\varepsilon$ ) number, and integrating over $\Omega$, we find

$$
\begin{aligned}
\frac{d}{d t} \bar{\Gamma}(t)+2(1-\alpha \varepsilon) & \left\|\partial_{t} v(t)\right\|_{L^{2}}^{2}+2 \alpha\left\|\nabla_{x} v(t)\right\|_{L^{2}}^{2}= \\
& =4\left(h(t),\left(-\Delta_{x}\right)^{-1} \partial_{t} h(t)\right)_{L^{2}}+2\left(\alpha h(t)-\partial_{t} h(t), v(t)\right)_{L^{2}}
\end{aligned}
$$

where

$$
\begin{aligned}
(\mathrm{A} .10) \quad \bar{\Gamma}(t):= & \varepsilon\left\|\partial_{t} v(t)\right\|_{L^{2}}^{2}+\left\|\nabla_{x} v(t)\right\|_{L^{2}}^{2}+ \\
& +\alpha\|v(t)\|_{L^{2}}^{2}-2(h(t), v(t))_{L^{2}}+2 \varepsilon \alpha\left(v(t), \partial_{t} v(t)\right)_{L^{2}}+2\|h(t)\|_{H^{-1}}^{2} .
\end{aligned}
$$

Moreover, for a sufficiently small $\alpha>0$, we have, obviously

$$
\begin{aligned}
& C_{1}^{-1}\left(\varepsilon\left\|\partial_{t} v(t)\right\|_{L^{2}}^{2}+\left\|\nabla_{x} v(t)\right\|_{L^{2}}^{2}+\|h(t)\|_{H^{-1}}^{2}\right) \leq \bar{\Gamma}(t) \leq \\
& \quad \leq C_{1}\left(\varepsilon\left\|\partial_{t} v(t)\right\|_{L^{2}}^{2}+\left\|\nabla_{x} v(t)\right\|_{L^{2}}^{2}+\|h(t)\|_{H^{-1}}^{2}\right),
\end{aligned}
$$

where the constant $C_{1}$ is independent of $\varepsilon$. Thus, (A.9) implies the following estimate:

$$
\frac{d}{d t} \bar{\Gamma}(t)+\alpha^{\prime} \bar{\Gamma}(t)+\alpha\left\|\partial_{t} v(t)\right\|_{L^{2}}^{2} \leq C\left(\|h(t)\|_{H^{-1}}+\left\|\partial_{t} h(t)\right\|_{H^{-1}}\right) \bar{\Gamma}(t)^{1 / 2}
$$

where the positive constants $\alpha^{\prime}$ and $C$ are independent of $\varepsilon$. In order to deduce a proper estimate on $\bar{\Gamma}(t)$ from (A.12), we need the following lemma. 
Lemma A.1. Let the positive function $z \in C^{1}\left(\mathbb{R}_{+}\right)$satisfy the following differential inequality

$$
\frac{d}{d t} z(t)+\alpha z(t) \leq H(t)[z(t)]^{1 / 2}+G(t)
$$

for some positive constant $\alpha$ and nonnegative functions $H$ and $G$. Then, the following estimate is valid

$$
\begin{array}{r}
\text { (A.14) } z(T) \leq z(0) e^{-\alpha T+1}+C\left(\int_{0}^{T} e^{-\alpha(T-s) / 2} H(s) d s\right)^{2}+ \\
+C \int_{0}^{T} e^{-\alpha(T-s)} G(s) d s,
\end{array}
$$

where the constant $C$ depends only on $\alpha$.

Proof of Lemma A.1. We set $\Lambda(T):=\int_{0}^{T} e^{-\alpha(T-t) / 2} H(t) d t$. Then, estimating the term $H(t)[z(t)]^{1 / 2}$ as follows:

$$
H(t)[z(t)]^{1 / 2} \leq e^{-\alpha(T-t) / 2} \Lambda(T)^{-1} H(t) z(t)+\Lambda(T) e^{\alpha(T-t) / 2} H(t),
$$

we deduce the following inequality:

$$
\frac{d}{d t} z(t)+\left(\alpha-\Lambda(T)^{-1} e^{-\alpha(T-t) / 2} H(t)\right) z(t) \leq G(t)+e^{\alpha(T-t) / 2} \Lambda(T) H(t) .
$$

Integrating inequality (A.15), we obtain

$$
\begin{aligned}
& z(T) e^{\alpha T-1}-z(0) \leq \\
& \leq \int_{0}^{T} e^{\alpha t-\Lambda(T)^{-1} \int_{0}^{t} e^{-\alpha(T-s) / 2} H(s) d s}\left(G(t)+\Lambda(T) e^{\alpha(T-t) / 2} H(t)\right) d t \leq \\
& \quad \leq \int_{0}^{T} e^{\alpha t} G(t) d t+\Lambda(T) e^{\alpha T} \int_{0}^{T} e^{-\alpha(T-t) / 2} H(t) d t .
\end{aligned}
$$

Dividing this inequality by $e^{\alpha T-1}$, we deduce estimate (A.14) and finish the proof of Lemma A.1.

Applying now Lemma A.1 to inequality (A.12) and using (A.11), we obtain

$$
\begin{aligned}
& \varepsilon\left\|\partial_{t} v(t)\right\|_{L^{2}}^{2}+\|v(t)\|_{H^{1}}^{2}+\|h(t)\|_{H^{-1}}^{2} \leq \\
& \leq C e^{-\alpha^{\prime} t}\left(\varepsilon\left\|\partial_{t} v(0)\right\|_{L^{2}}^{2}+\|v(0)\|_{H^{1}}^{2}+\|h(0)\|_{H^{-1}}^{2}\right)+ \\
& \quad+C\left(\int_{0}^{t} e^{-\alpha^{\prime}(t-s) / 2}\left(\|h(s)\|_{H^{-1}}+\left\|\partial_{t} h(s)\right\|_{H^{-1}}\right) d s\right)^{2}
\end{aligned}
$$


where the constants $C$ and $\alpha^{\prime}$ are independent of $\varepsilon$. We now derive the desired estimate for the integral of $\left\|\partial_{t} v(s)\right\|_{L^{2}}^{2}$. To this end, we multiply inequality (A.12) by $e^{-\alpha^{\prime}(T-t)}$, integrate over $[0, T]$ and use the obvious fact that

$$
\int_{0}^{T} e^{-\alpha^{\prime}(T-t)}\left[z^{\prime}(t)+\alpha^{\prime} z(t)\right] d t=z(T)-z(0) e^{-\alpha^{\prime} T} .
$$

Then, according to (A.11), (A.12) and (A.16), we have

$$
\begin{gathered}
\alpha \int_{0}^{T} e^{-\alpha^{\prime}(T-t)}\left\|\partial_{t} v(t)\right\|_{L^{2}}^{2} d t \leq \bar{\Gamma}(0) e^{-\alpha^{\prime} T}-\bar{\Gamma}(T)+ \\
+C \int_{0}^{T} e^{-\alpha^{\prime}(T-t)} H(t) \bar{\Gamma}(t)^{1 / 2} d t \leq \\
\leq \bar{\Gamma}(0) e^{-\alpha^{\prime} T}+C \int_{0}^{T} e^{-\alpha^{\prime}(T-t) / 2} H(t) \cdot e^{-\alpha^{\prime}(T-t) / 2-\alpha^{\prime} t / 2}[\bar{\Gamma}(0)]^{1 / 2} d t+ \\
+C \int_{0}^{T} e^{-\alpha^{\prime}(T-t)} H(t) \int_{0}^{t} e^{-\alpha^{\prime}(T-s) / 2} H(s) d s d t \leq \\
\leq C_{1} e^{-\alpha^{\prime} T} \bar{\Gamma}(0)+C_{1}\left(\int_{0}^{T} e^{-\alpha^{\prime}(T-t)} H(t) d t\right)^{2}
\end{gathered}
$$

where $H(t):=\|h(t)\|_{H^{-1}}+\left\|\partial_{t} h(t)\right\|_{H^{-1}}$. Estimate (A.17) gives the desired estimate for the integral of $\left\|\partial_{t} v(t)\right\|_{L^{2}}^{2}$. Thus, it only remains to obtain the estimate for the $H^{-1}$-norm of $\partial_{t} v(t)$. Moreover, due to Proposition A.1, we can assume that $\xi_{v}(0)=0$. We differentiate equation (A.1) with respect to $t$, multiply the equation that we obtain by $\left(-\Delta_{x}\right)^{-1}\left(2 \varepsilon \partial_{t}^{2} v(t)+\partial_{t} v(t)\right)$ and integrate over $\Omega$. Then, we have

$$
\begin{array}{rl}
\frac{d}{d t} \Gamma_{v}(t)+\varepsilon\left\|\partial_{t}^{2} v(t)\right\|_{H^{-1}}^{2}+\| \partial_{t} & v(t) \|_{L^{2}}^{2}= \\
& =\left(\partial_{t} h(t),\left(-\Delta_{x}\right)^{-1}\left(2 \varepsilon \partial_{t}^{2} v(t)+\partial_{t} v(t)\right)\right)_{L^{2}}
\end{array}
$$

where

$$
\begin{aligned}
\Gamma_{v}(t):=\varepsilon\left(\partial_{t} v(t),\left(-\Delta_{x}\right)^{-1} \partial_{t}^{2} v(t)\right)_{L^{2}}+ & \frac{1}{2}\left\|\partial_{t} v(t)\right\|_{H^{-1}}^{2}+ \\
& +\varepsilon\left\|\partial_{t} v(t)\right\|_{L^{2}}^{2}+\varepsilon^{2}\left\|\partial_{t}^{2} v(t)\right\|_{H^{-1}}^{2}
\end{aligned}
$$

We note that

$$
\begin{aligned}
C^{-1}\left(\varepsilon^{2}\left\|\partial_{t}^{2} v(t)\right\|_{H^{-1}}^{2}\right. & \left.+\varepsilon\left\|\partial_{t} v(t)\right\|_{L^{2}}^{2}+\left\|\partial_{t} v(t)\right\|_{H^{-1}}^{2}\right) \leq \Gamma_{v}(t) \leq \\
& \leq C\left(\varepsilon^{2}\left\|\partial_{t}^{2} v(t)\right\|_{H^{-1}}^{2}+\varepsilon\left\|\partial_{t} v(t)\right\|_{L^{2}}^{2}+\left\|\partial_{t} v(t)\right\|_{H^{-1}}^{2}\right)
\end{aligned}
$$


where the positive constant $C$ is independent of $\varepsilon$ and, consequently, for sufficiently small $\varepsilon>0,($ A.18) yields

$$
\frac{d}{d t} \Gamma_{v}(t)+\alpha \Gamma_{v}(t) \leq C_{1}\left\|\partial_{t} h(t)\right\|_{H^{-1}} \Gamma_{v}(t)^{1 / 2},
$$

where the positive constants $\alpha$ and $C_{1}$ are independent of $\varepsilon$. Applying Lemma A.1 to the differential inequality (A.21), we obtain

$$
\begin{aligned}
& \varepsilon^{2}\left\|\partial_{t}^{2} v(t)\right\|_{H^{-1}}^{2}+\varepsilon\left\|\partial_{t} v(t)\right\|_{L^{2}}^{2}+\left\|\partial_{t} v(t)\right\|_{H^{-1}}^{2} \leq \\
& \quad \leq C e^{-\alpha t} \varepsilon^{2}\left\|\partial_{t}^{2} v(0)\right\|_{H^{-1}}^{2}+C\left(\int_{0}^{t} e^{-\alpha(t-s)}\left\|\partial_{t} h(s)\right\|_{H^{-1}} d s\right)^{2} .
\end{aligned}
$$

Here, we have also used the fact that $\xi_{v}(0)=0$. It remains to note that equation (A.1) implies that $\varepsilon \partial_{t}^{2} v(0)=h(0)$ and, consequently, the desired estimate for the $H^{-1}$-norm of $\partial_{t} v(t)$ is an immediate consequence of (A.22). This finishes the proof of Proposition A.2.

Acknowledgments : The first and the third authors have been supported by Italian MIUR-COFIN project "Problemi di frontiera libera nelle scienze applicate". The fourth author has been partially supported by INTAS project no. 00-899 and CRDF grant no. 10545 .

\section{REFERENCES}

[BV] A.V. Babin and M.I. Vishik, Attractors of evolution equations, Nauka, Moscow, 1989; English translation: North-Holland, Amsterdam, 1992.

[BrS] M. Brokate and J. Sprekels, Hysteresis and phase transitions, Springer, New York, 1996.

[Ca] G. Caginalp, An analysis of a phase field model of a free boundary, Arch. Rational Mech. Anal. 92, 205-245, 1986.

[CV] V.V. Chepyzhov and M.I. Vishik, Attractors for equations of mathematical physics, Amer. Math. Soc., Providence, RI, 2002.

[EFNT] A. Eden, C. Foias, B. Nicolaenko and R. Temam, Exponential attractors for dissipative evolution equations, John-Wiley, New York, 1994.

[EfMZ1] M. Efendiev, A. Miranville and S. Zelik, Exponential attractors for a nonlinear reaction-diffusion system in $\mathbb{R}^{3}$, C. R. Acad. Sci. Paris, Sér. I 330, 713-718, 2000.

[EfMZ2] M. Efendiev, A. Miranville and S. Zelik, Exponential attractors for a singularly perturbed Cahn-Hilliard system, Math. Nachr. 272, 11-31, 2004.

[FGMZ] P. Fabrie, C. Galusinski, A. Miranville and S. Zelik, Uniform exponential attractors for a singularly perturbed damped wave equation, Discrete Contin. Dynam. Systems 10 (1-2), 211-238, 2004.

[GrP1] M. Grasselli and V. Pata, Existence of a universal attractor for a parabolichyperbolic phase-field system, Adv. Math. Sci. Appl. 13 (2), 443-459, 2003. 
[GrP2] M. Grasselli and V. Pata, Asymptotic behavior of a parabolic-hyperbolic problem, Comm. Pure Appl. Anal. 3 (4), 849-881, 2004.

[H] J.K. Hale, Asymptotic behavior of dissipative systems, Math. Surveys Monogr., Vol. 25, Amer. Math. Soc., Providence, RI, 1987.

[L] O.A. Ladyzhenskaya, Attractors for semigroups and evolution equations, Lincei Lectures, Cambridge University Press, Cambridge, 1991.

[LSU] O.A. Ladyzhenskaya, V.A. Solonnikov and N.N. Ural'ceva, Linear and quasilinear equations of parabolic type, Transl. Math. Monogr., Amer. Math. Soc., Providence, RI, 1968.

[LyV] L.A. Lyusternik and M.I. Vishik, Regular degeneration and boundary layer for linear differential equations with small parameter, Amer. Math. Soc. Transl. Ser. 2 20, 239-364, 1962.

[Mie] A. Mielke Über maximale L(p)-Regularität für Differentialgleichungen in Banach- und Hilbert-Räumen, Math. Ann. 277 (1), 121-133, 1987.

[MZ1] A. Miranville and S. Zelik, Robust exponential attractors for singularly perturbed phase-field type equations, Electron. J. Differential Equations 63, 1-28, 2002.

[MZ2] A. Miranville and S. Zelik, Robust exponential attractors for Cahn-Hilliard type equations with singular potentials, Math. Models Appl. Sci. 27 (5), 545-582, 2004.

[RDN] H.R. Rotstein, A.I. Domoshnitsky and A. Nepomnyashchy, Front motion for phase transitions with linear memory, Phys. D 146, 137-149, 2000.

[T] R. Temam, Infinite-dimensional dynamical systems in mechanics and physics, 2d ed., Springer-Verlag, New York, 1997.

[Z] S. Zelik, Asymptotic regularity of solutions of singularly perturbed damped wave equations with supercritical nonlinearities, Discrete Contin. Dynam. Systems 11 (2-3), 351-392, 2004. 